\title{
PERTURBATION THEORY FOR THE NONLINEAR SCHRÖDINGER EQUATION WITH A RANDOM POTENTIAL
}

\author{
SHMUEL FISHMAN, YEVGENY KRIVOLAPOV, AND AVY SOFFER
}

\begin{abstract}
A perturbation theory for the Nonlinear Schrödinger Equation (NLSE) in 1D on a lattice was developed. The small parameter is the strength of the nonlinearity. For this purpose secular terms were removed and a probabilistic bound on small denominators was developed. It was shown that the number of terms grows exponentially with the order. The results of the perturbation theory are compared with numerical calculations. An estimate on the remainder is obtained and it is demonstrated that the series is asymptotic.
\end{abstract}

\section{INTRODUCTION}

We consider the problem of dynamical localization of waves in a Nonlinear Schrödinger Equation (NLSE) [1] with a random potential term on a lattice:

$$
i \partial_{t} \psi=-J[\psi(x+1)+\psi(x-1)]+\varepsilon_{x}^{\omega} \psi+\beta|\psi|^{2} \psi
$$

where $\psi=\psi(x, t), x \in \mathbb{Z}$; and $\left\{\varepsilon_{x}^{\omega}\right\}_{\omega \in \Omega}$ is a collection of i.i.d. random variables chosen from the set $\Omega$, with the probability measure $\mu\left(\varepsilon_{x}\right)$. It will be assumed that $\mu(\varepsilon(x))$ is continuous, bounded and of finite support and, additionally, symmetric, $\mu\left(\varepsilon_{x}\right)=\mu\left(-\varepsilon_{x}\right)$. We assume that exponential localization is known to take place for all the energies of the linear problem (when $\beta=0$ ). The decay rate $\gamma$ and the localization length, $\xi=1 / \gamma$, are given for the linear part of the model (1.1) by the Thouless formula [2]. In particular, if $\mu\left(\varepsilon_{x}\right)$ is a uniform distribution than as was found numerically (see Appendix), the function $\gamma(E)$ is unimodal.

The NLSE was derived for a variety of physical systems under some approximations. It was derived in classical optics where $\psi$ is the electric field by expanding the index of refraction in powers of the electric field keeping only the leading nonlinear term [3]. For Bose-Einstein Condensates (BEC), the NLSE is a mean field approximation where the density $\beta|\psi|^{2}$ approximates the interaction between the atoms. In this field the NLSE is known as the Gross-Pitaevskii Equation (GPE) $[4,5,6,7,8,9]$. Recently, it was rigorously established, for a large variety of interactions and of physical conditions, that the NLSE (or the GPE) is exact in the thermodynamic limit [10,11]. Generalized mean field theories, in which several mean-fields are used, were recently developed $[12,13]$. In the absence of randomness (1.1) is completely integrable. For repulsive nonlinearity $(\beta>0)$ an initially localized wavepacket spreads, while for attractive nonlinearity $(\beta<0)$ solitons are found typically [1].

It is well known that in $1 \mathrm{D}$ in the presence of a random potential and in the absence of nonlinearity $(\beta=0)$ with probability one all the states are exponentially

Date: January 23rd, 2009.

Key words and phrases. Anderson localization, NLSE, random potential, nonlinear Schrodinger, dynamical localization, diffusion, sub-diffusion. 
localized [14, 15, 16, 17]. Consequently, diffusion is suppressed and in particular a wavepacket that is initially localized will not spread to infinity. This is the phenomenon of Anderson localization. In $2 \mathrm{D}$ it is known heuristically from the scaling theory of localization $[18,16]$ that all the states are localized, while in higher dimensions there is a mobility edge that separates localized and extended states. This problem is relevant for experiments in nonlinear optics, for example disordered photonic lattices [19], where Anderson localization was found in presence of nonlinear effects as well as experiments on BECs in disordered optical lattices $[20,21,22,23,24,25,26,27,28]$. The interplay between disorder and nonlinear effects leads to new interesting physics [26, 27, 29, 30, 31, 32]. In spite of the extensive research, many fundamental problems are still open, and, in particular, it is not clear whether in one dimension (1D) Anderson localization can survive the effects of nonlinearities. This will be studied here.

A natural question is whether a wave packet that is initially localized in space will indefinitely spread for dynamics controlled by (1.1). A simple argument indicates that spreading will be suppressed by randomness. If unlimited spreading takes place the amplitude of the wave function will decay since the $L^{2}$ norm is conserved. Consequently, the nonlinear term will become negligible and Anderson localization will take place as a result of the randomness. Contrary to this intuition, based on the smallness of the nonlinear term resulting from the spread of the wave function, it is claimed that for the kicked-rotor a nonlinear term leads to delocalization if it is strong enough [33]. It is also argued that the same mechanism results in delocalization for the model (1.1) with sufficiently large $\beta$, while, for weak nonlinearity, localization takes place [33, 34]. Therefore, it is predicted in that work that there is a critical value of $\beta$ that separates the occurrence of localized and extended states. However, if one applies the arguments of [33, 34] to a variant of (1.1), results that contradict numerical solutions are found [35, 36]. Recently, it was rigorously shown that the initial wavepacket cannot spread so that its amplitude vanishes at infinite time, at least for large enough $\beta$ [37]. It does not contradict spreading of a fraction of the wavefunction. Indeed, subdiffusion was found in numerical experiments [33, 37, 38]. In different works [38, 39, 40] sub-diffusion was reported for all values of $\beta$, but with a different power of the time dependence (compared with Ref. [33]). It was also argued that nonlinearity may enhance discrete breathers [31, 32]. In conclusion, it is not clear what is the long time behavior of a wave packet that is initially localized, if both nonlinearity and disorder are present. This is the main motivation for the present work. Since heuristic arguments and numerical simulations produce conflicting results, rigorous statements are required for further progress.

More precisely, the question of dynamical localization can be rigorously formulated as follows: assume the initial state is, $\psi(x, 0) \equiv u_{0}(x)$, where $u_{0}(x)$ is an eigenstate of the linear part of (1.1) which is localized near $x=0$. Then for any $0<\delta<1$, one has to prove that with probability $1-\delta$ (on the space of the potentials)

$$
\sup _{x, t}\left|e^{\nu|x|} \psi(x, t)\right|<M_{\delta}<\infty
$$

for some $\nu>0$.

Rigorous results on dynamical localization for the linear case are well known $[41,42,43]$. However, the nonlinear problem turns out to be very difficult to 
handle, even numerically. Consider the case of small $\beta$. There are two possible mechanisms for destruction of the localization due to nonlinearity.

One way of spreading is to spread into many random places with increasing number of them. In that case, due to conservation of the normalization of the solution, the solution becomes small. But then, the nonlinear term becomes less and less important and we expect the linear theory to take over and lead to localization. While this argument sounds plausible there is no proof along this lines.

The second way of spreading is in a few fixed number of spikes that hop randomly to infinity. In this case, the nonlinear term is always relevant. It is this (possible) process that makes the proof of localization in the nonlinear case so elusive. It also precludes a quick numerical analysis of the problem: it may take exponentially long time to see the hoping.

Rigorous results in this direction are of preliminary nature: In [44] it was shown that dynamical localization holds for the linear problem perturbed by a periodic in time and exponentially localized in space small linear perturbation. In [45] the above result was extended to a quasiperiodic in time perturbation. Such perturbations mimic the nonlinear term:

$$
|\psi|^{2} \rightarrow\left|\sum_{j} c_{j} u_{j}(x) e^{i E_{j} t}\right|^{2}
$$

where $u_{j}$ are the eigenfunctions of the linear problem with energies $E_{j}$. However in other situations time dependent terms may result in delocalization [46, 47]. Using normal form transformations Wang and Zhang [48] studied the limit of strong disorder and weak nonlinearity, namely, $\epsilon=J+\beta$, small. For initial wavefunctions with tails of weight $\delta$ starting from point $j_{0}$, they have proved, that the wavefunction spreads as following. There exist $C=C(A)>0$ and $\epsilon(A)>0$ and $K=K(A)>A^{2}$ such that for all $t \leq(\delta / C) \epsilon^{-A}$ the weight of the tails of the spreaded wavefunction starting from $j=j_{0}+K$ is less that $2 \delta$. On the basis of this result they have conjectured that the spread of the wave function is at most logarithmic in $t$.

Furthermore, it can be shown that NLSE has stationary solutions

$$
E \psi_{E}=\left(-\partial_{x x}+\varepsilon_{x}^{\omega}\right) \psi_{E}+\beta\left|\psi_{E}\right|^{2} \psi_{E}
$$

which are exponentially localized for almost all $E$ with a localization length that is identical to the one of the linear problem [49, 50, 51, 52, 53].

In our previous work [54] we have developed a perturbation theory in $\beta$. By considering the first order expansion we have proved that for times of order $O\left(\beta^{-2}\right)$ the solution of (1.1) remains exponentially localized. A result of similar nature for a nonlinear equation of a different structure was obtained in [55]. In the current work we consider an expansion of any order, $N$, in $\beta$. This expansion enables in principle the calculation of the solution to any order in $N$. A bound on the error can be computed using only propreties of the linear problem $(\beta=0)$. Therefore this work has the potential to develop into a method for solution of some type of nonlinear differential equations. In Section 2 we construct the solution as a series in the eigenfunctions of the linear problem. Standard perturbation theory for the coefficients does not apply: we encounter small divisor problems and secular terms (formally infinite). Removing the secular terms requires the "renormalization" of the original linear Hamiltonian by shifting the energies (Section 3). The estimates 
of the small divisor terms are performed in the spirit of the work of AizenmanMolchanov (A-M) [56]. In Section 4 the entropy problem is resolved by bounding an appropriate recursive relation. A general probabilistic bound on the terms of the perturbation theory is derived in Section 5 and the quality of the perturbation theory is tested in Section 6. In Section 7 the remainder terms are controlled by a bootstrap argument. The results are summarized in Section 8 and the open problems are listed there.

In summary, in this work a perturbation theory for (1.1) in powers of $\beta$ was developed and bounds on the various terms were obtained. The work is only partly rigorous. In some parts it relies on Conjectures that we test numerically.

\section{Organization of The Perturbation theOry}

Our goal is to analyze the nonlinear Schrödinger equation

$$
i \partial_{t} \psi=H_{0} \psi+\beta|\psi|^{2} \psi
$$

where $H_{0}$ is the Anderson Hamiltonian,

$$
H_{0} \psi(x)=-J[\psi(x+1)+\psi(x-1)]+\varepsilon_{x} \psi(x) .
$$

We assume throughout the paper that $H_{0}$ satisfies the conditions for localization, namely, for almost all the realizations, $\omega$, of the disordered potential, all the eigenstates of $H_{0}, u_{m}$, are exponentially localized and have an envelope of the form of

$$
\left|u_{m}(x)\right| \leq D_{\omega, \varepsilon} e^{\varepsilon\left|x_{m}\right|} e^{-\gamma\left|x-x_{m}\right|},
$$

where $\varepsilon>0, x_{m}$ is the localization center which will be defined at the next subsection, $\gamma$ is the inverse of the localization length, $\xi=\gamma^{-1}$, and $D_{\omega, \varepsilon}$ is a constant dependent on $\varepsilon$ and the realization of the disordered potential [57, 58] (better estimates were proven recently in $[59,60])$. It is of importance that $D_{\omega, \varepsilon}$ does not depend on the energy of the state. In the present work only realizations $\omega$, where

$$
\left|D_{\omega, \varepsilon}\right| \leq D_{\delta, \varepsilon}<\infty
$$

are considered. This is satisfied for a set of a measure $1-\delta$, since (2.3) is false only for a measure zero of potentials.

2.1. Assignment of eigenfunctions to sites. It is tempting to assign eigenfunctions to sites by their maxima, namely, $u_{E_{i}}$ is the eigenfunction with energy $E_{i}$ and a maximum at site $i$. This assignment is very unstable with respect to the change of realizations. This is due to the fact that the point where the maximum is found, which is sometimes called the localization center, may change as a result of a very small change in the on-site energies $\left\{\varepsilon_{x}\right\}$. To avoid this, the assignment is defined as the center of mass [61],

$$
x_{E}=\sum_{x} x\left|u_{E_{i}}(x)\right|^{2}
$$

Definition 1. The state $u_{E_{i}}$ is assigned to site $i$ if $i=\left[x_{E}\right]$. If several states are assigned to the same site we order them by energy. 
2.2. The perturbation expansion. The wavefunction can be expanded using the eigenstates of $H_{0}$ as

$$
\psi(x, t)=\sum_{m} c_{m}(t) e^{-i E_{m} t} u_{m}(x) .
$$

For the nonlinear equation the dependence of the expansion coefficients, $c_{m}(t)$, is found by inserting this expansion into (2.1), resulting in

$$
\begin{aligned}
i \partial_{t} \sum_{m} c_{m} e^{-i E_{m} t} u_{m}(x) & =H_{0} \sum_{m} c_{m} e^{-i E_{m} t} u_{m}(x) \\
& +\beta\left|\sum_{m} c_{m} e^{-i E_{m} t} u_{m}(x)\right|^{2} \sum_{m_{3}} c_{m_{3}} e^{-i E_{m_{3}} t} u_{m_{3}}(x) .
\end{aligned}
$$

Multiplying by $u_{n}(x)$ and integrating gives

$$
i \partial_{t} c_{n}=\beta \sum_{m_{1}, m_{2}, m_{3}} V_{n}^{m_{1} m_{2} m_{3}} c_{m_{1}}^{*} c_{m_{2}} c_{m_{3}} e^{i\left(E_{m_{1}}+E_{n}-E_{m_{2}}-E_{m_{3}}\right) t}
$$

where $V_{n}^{m_{1} m_{2} m_{3}}$ is an overlap sum

$$
V_{n}^{m_{1} m_{2} m_{3}}=\sum_{x} u_{n}(x) u_{m_{1}}(x) u_{m_{2}}(x) u_{m_{3}}(x) .
$$

By definition $V_{n}^{m_{1} m_{2} m_{3}}$ is symmetric with respect to an interchange of any two indices. Additionally, since the $u_{n}(x)$ are exponentially localized around $x_{n}, V_{n}^{m_{1} m_{2} m_{3}}$ is not negligible only when the interval,

$$
\delta m \equiv \max \left[x_{n}, x_{m_{i}}\right]-\min \left[x_{n}, x_{m_{i}}\right],
$$

is of the order of the localization length, around $x_{n}$,

$$
\begin{aligned}
\left|V_{n}^{m_{1} m_{2} m_{3}}\right| & \leq D_{\delta, \varepsilon}^{4} e^{\varepsilon\left(\left|x_{n}\right|+\left|x_{m_{1}}\right|+\left|x_{m_{2}}\right|+\left|x_{m_{3}}\right|\right)} \sum_{x} \cdot e^{-\gamma\left(\left|x-x_{n}\right|+\left|x-x_{m_{1}}\right|+\left|x-x_{m_{2}}\right|+\left|x-x_{m_{3}}\right|\right)} \\
& \leq D_{\delta, \varepsilon}^{4} e^{\varepsilon\left(\left|x_{n}\right|+\left|x_{m_{1}}\right|+\left|x_{m_{2}}\right|+\left|x_{m_{3}}\right|\right)} e^{-\frac{\left(\gamma-\varepsilon^{\prime}\right)}{3}\left(\left|x_{n}-x_{m_{1}}\right|+\left|x_{n}-x_{m_{2}}\right|+\left|x_{n}-x_{m_{3}}\right|\right)_{\times}} \\
& \times \sum_{x} \cdot e^{-\varepsilon^{\prime}\left(\left|x-x_{n}\right|+\left|x-x_{m_{1}}\right|+\left|x-x_{m_{2}}\right|+\left|x-x_{m_{3}}\right|\right)} \\
& \leq V_{\delta}^{\varepsilon, \varepsilon^{\prime}} e^{\varepsilon\left(\left|x_{n}\right|+\left|x_{m_{1}}\right|+\left|x_{m_{2}}\right|+\left|x_{m_{3}}\right|\right)} e^{-\frac{1}{3}\left(\gamma-\varepsilon^{\prime}\right)\left(\left|x_{n}-x_{m_{1}}\right|+\left|x_{n}-x_{m_{2}}\right|+\left|x_{n}-x_{m_{3}}\right|\right)} .
\end{aligned}
$$

Here we have used the triangle inequality

$$
\begin{aligned}
& \left(\left|x-x_{n}\right|+\left|x-x_{m_{1}}\right|\right)+\left(\left|x-x_{n}\right|+\left|x-x_{m_{2}}\right|\right)+ \\
& +\left(\left|x-x_{n}\right|+\left|x-x_{m_{3}}\right|\right) \geq\left|x_{n}-x_{m_{1}}\right|+\left|x_{n}-x_{m_{2}}\right|+\left|x_{n}-x_{m_{3}}\right|
\end{aligned}
$$

to obtain the second line. Our objective is to develop a perturbation expansion of the $c_{m}(t)$ in powers of $\beta$ and to calculate them order by order in $\beta$. The required expansion is

$$
c_{n}(t)=c_{n}^{(0)}+\beta c_{n}^{(1)}+\beta^{2} c_{n}^{(2)}+\cdots+\beta^{N-1} c_{n}^{(N-1)}+\beta^{N} Q_{n},
$$

where the expansion is till order $(N-1)$ and $Q_{n}$ is the remainder term. We will assume the initial condition

$$
c_{n}(t=0)=\delta_{n 0} .
$$


The equations for the two leading orders are presented in what follows. The leading order is

$$
c_{n}^{(0)}=\delta_{n 0}
$$

The equation for the first order is

$$
i \partial_{t} c_{n}^{(1)}=\sum_{m_{1}, m_{2}, m_{3}} V_{n}^{m_{1} m_{2} m_{3}} c_{m_{1}}^{*(0)} c_{m_{2}}^{(0)} c_{m_{3}}^{(0)} e^{i\left(E_{n}+E_{m_{1}}-E_{m_{2}}-E_{m_{3}}\right) t}=V_{n}^{000} e^{i\left(E_{n}-E_{0}\right) t}
$$

and its solution is

$$
c_{n}^{(1)}=V_{n}^{000}\left(\frac{1-e^{i\left(E_{n}-E_{0}\right) t}}{E_{n}-E_{0}}\right) .
$$

The resulting equation for the second order is

$$
\begin{aligned}
i \partial_{t} c_{n}^{(2)} & =\sum_{m_{1}, m_{2}, m_{3}} V_{n}^{m_{1} m_{2} m_{3}} c_{m_{1}}^{*(1)} c_{m_{2}}^{(0)} c_{m_{3}}^{(0)} e^{i\left(E_{n}+E_{m_{1}}-E_{m_{2}}-E_{m_{3}}\right) t}+ \\
& +2 \sum_{m_{1}, m_{2}, m_{3}} V_{n}^{m_{1} m_{2} m_{3}} c_{m_{1}}^{*(0)} c_{m_{2}}^{(1)} c_{m_{3}}^{(0)} e^{i\left(E_{n}+E_{m_{1}}-E_{m_{2}}-E_{m_{3}}\right) t} .
\end{aligned}
$$

Substitution of the lower orders yields

$$
\begin{aligned}
i \partial_{t} c_{n}^{(2)} & =\sum_{m} V_{n}^{m 00} V_{m}^{000}\left[\left(\frac{1-e^{-i\left(E_{m}-E_{0}\right) t}}{E_{m}-E_{0}}\right) e^{i\left(E_{n}+E_{m}-2 E_{0}\right) t}+\right. \\
& \left.+2\left(\frac{1-e^{i\left(E_{m}-E_{0}\right) t}}{E_{m}-E_{0}}\right) e^{i\left(E_{n}-E_{m}\right) t}\right] \\
& =\sum_{m} \frac{V_{n}^{m 00} V_{m}^{000}}{E_{m}-E_{0}}\left[\left(e^{i\left(E_{n}+E_{m}-2 E_{0}\right) t}-e^{i\left(E_{n}-E_{0}\right) t}\right)+\right. \\
& \left.+2\left(e^{i\left(E_{n}-E_{m}\right) t}-e^{i\left(E_{n}-E_{0}\right) t}\right)\right] \\
& =\sum_{m} \frac{V_{n}^{m 00} V_{m}^{000}}{E_{m}-E_{0}}\left[e^{i\left(E_{n}+E_{m}-2 E_{0}\right) t}-3 e^{i\left(E_{n}-E_{0}\right) t}+2 e^{i\left(E_{n}-E_{m}\right) t}\right] .
\end{aligned}
$$

We notice that divergence of this expansion for any value of $\beta$ may result from three major problems: the secular terms problem, the entropy problem (i.e., factorial proliferation of terms), and the small denominators problem.

\section{Elimination of Secular terms}

We first show how to derive the equations for $c_{n}(t)$ where the secular terms are eliminated.

Proposition 2. To each order in $\beta, \psi(x, t)$ can be expanded as

$$
\psi(x, t)=\sum_{n} c_{n}(t) e^{-i E_{n}^{\prime} t} u_{n}(x)
$$

with

$$
E_{n}^{\prime} \equiv E_{n}^{(0)}+\beta E_{n}^{(1)}+\beta^{2} E_{n}^{(2)}+\cdots
$$

and $E_{n}^{(0)}$ are the eigenvalues of $H_{0}$, in such a way that there are no secular terms to any given order. The $E_{n}^{\prime}$ are called the renormalized energies. 
Here we first develop the general scheme for the elimination of the secular terms and then demonstrate the construction of $E_{n}^{\prime}$ when the $c_{n}(t)$ are calculated to the second order in $\beta$ (see $3.19,3.18$ ).

Inserting the expansion into (2.1) yields

$$
\begin{aligned}
i \sum_{m}\left[\partial_{t} c_{m}-\right. & \left.i E_{m}^{\prime} c_{m}\right] e^{-i E_{m}^{\prime} t} u_{m}(x)=\sum_{m} E_{m}^{(0)} c_{m} e^{-i E_{m}^{\prime} t} u_{m}(x)+ \\
& +\beta \sum_{m_{1} m_{2} m_{3}} c_{m_{1}}^{*} c_{m_{2}} c_{m_{3}} e^{i\left(E_{m_{1}}^{\prime}-E_{m_{2}}^{\prime}-E_{m_{3}}^{\prime}\right) t} u_{m_{1}}(x) u_{m_{2}}(x) u_{m_{3}}(x) .
\end{aligned}
$$

Multiplication by $u_{n}(x)$ and integration gives

$$
i \partial_{t} c_{n}=\left(E_{n}^{(0)}-E_{n}^{\prime}\right) c_{n}+\beta \sum_{m_{1} m_{2} m_{3}} V_{n}^{m_{1} m_{2} m_{3}} c_{m_{1}}^{*} c_{m_{2}} c_{m_{3}} e^{i\left(E_{n}^{\prime}+E_{m_{1}}^{\prime}-E_{m_{2}}^{\prime}-E_{m_{3}}^{\prime}\right) t}
$$

where the $V_{n}^{m_{1} m_{2} m_{3}}$ are given by (2.9). Following (2.13) we expand $c_{n}$ in orders of $\beta$, namely,

$$
c_{n}=c_{n}^{(0)}+\beta c_{n}^{(1)}+\beta^{2} c_{n}^{(2)}+\cdots, .
$$

Inserting this expansion into (3.4) and comparing the powers of $\beta$ without expanding the exponent, produces the following equation for the $k-t h$ order

$$
\begin{aligned}
i \partial_{t} c_{n}^{(k)} & =-\sum_{s=0}^{k-1} E_{n}^{(k-s)} c_{n}^{(s)}+ \\
& +\sum_{m_{1} m_{2} m_{3}} V_{n}^{m_{1} m_{2} m_{3}}\left[\sum_{r=0}^{k-1} \sum_{s=0}^{k-1-r} \sum_{l=0}^{k-1-r-s} c_{m_{1}}^{(r) *} c_{m_{2}}^{(s)} c_{m_{3}}^{(l)}\right] e^{i\left(E_{n}^{\prime}+E_{m_{1}}^{\prime}-E_{m_{2}}^{\prime}-E_{m_{3}}^{\prime}\right) t} .
\end{aligned}
$$

Note that the exponent is of order $O(1)$ in $\beta$, and therefore we may choose not to expand it in powers of $\beta$. However, it generates an expansion where both $E_{m}^{(l)}$ and $c_{n}^{(k)}$ depend on $\beta$. For the expansion (2.13) to be valid, both $E_{m}^{(l)}$ and $c_{n}^{(k)}$ should be $O(1)$ in $\beta$, this is satisfied since the RHS of (3.6) contains only $c_{n}^{(r)}$ such that $r<k$. Namely, this equation gives each order in terms of the lower ones, with the initial condition of $c_{n}^{(0)}(t)=\delta_{n 0}$. Solution of $k$ equations (3.6) gives the solution of the differential equation (3.4) to order $k$. Since, the exponent in (3.6) is of order $O(1)$ in $\beta$ we can select its argument to be of any order in $\beta$. However, for the removal of the secular terms, as will be explained bellow, it is instructive to set the order of the argument to be $k-1$, as the higher orders were not calculated at this stage. Secular terms are created when there are time independent terms in the RHS of the equation above. We eliminate those terms by using the first two terms in the first summation on the RHS. We make use of the fact that $c_{n}^{(0)}=\delta_{n 0}$ and $c_{n}^{(1)}$ can be easily determined (see $(3.9,3.12)$ ), and used to calculate $E_{n=0}^{(k)}$ and $E_{n \neq 0}^{(k-1)}$ that eliminate the secular terms in the equation for $c_{n}^{(k)}$, that is

$$
E_{n}^{(k)} c_{n}^{(0)}+E_{n}^{(k-1)} c_{n}^{(1)}=E_{n}^{(k)} \delta_{n 0}+E_{n}^{(k-1)}\left(1-\delta_{n 0}\right) \frac{V_{n}^{000}}{E_{n}^{\prime}-E_{0}^{\prime}},
$$


where only the time-independent part of $c_{n}^{(1)}$ was used. In other words, we choose $E_{n}^{(k)}$ and $E_{n \neq 0}^{(k-1)}$ so that the time-independent terms on the RHS of (3.6) are eliminated. $E_{0}^{(k)}$ will eliminate all secular terms with $n=0$, and $E_{n}^{(k-1)}$ will eliminate all secular terms with $n \neq 0$. In the following, we will demonstrate this procedure for the first two orders, and calculate $c_{n}^{(1)}$, and obtain an equation for $c_{n}^{(2)}$.

In the first order of the expansion in $\beta$ we obtain

$$
\begin{aligned}
i \partial_{t} c_{n}^{(1)} & =-E_{n}^{(1)} c_{n}^{(0)}+\sum_{m_{1} m_{2} m_{3}} V_{n}^{m_{1} m_{2} m_{3}} c_{m_{1}}^{*(0)} c_{m_{2}}^{(0)} c_{m_{3}}^{(0)} e^{i\left(E_{n}^{\prime}+E_{m_{1}}^{\prime}-E_{m_{2}}^{\prime}-E_{m_{3}}^{\prime}\right) t} \\
& =-E_{n}^{(1)} \delta_{n 0}+V_{n}^{000} e^{i\left(E_{n}^{\prime}-E_{0}^{\prime}\right) t} .
\end{aligned}
$$

For $n=0$ the equation produces a secular term

$$
\begin{aligned}
i \partial_{t} c_{0}^{(1)} & =-E_{0}^{(1)}+V_{0}^{000} \\
c_{0}^{(1)} & =i t \cdot\left(E_{0}^{(1)}-V_{0}^{000}\right) .
\end{aligned}
$$

Setting

$$
E_{0}^{(1)}=V_{0}^{000}
$$

will eliminate this secular term and gives

$$
c_{0}^{(1)}=0
$$

For $n \neq 0$ there are no secular terms in this order, therefore finally

$$
c_{n}^{(1)}=\left(1-\delta_{n 0}\right) V_{n}^{000}\left(\frac{1-e^{i\left(E_{n}^{\prime}-E_{0}^{\prime}\right) t}}{E_{n}^{\prime}-E_{0}^{\prime}}\right),
$$

where to this order $E_{n}^{\prime}=E_{n}$ and $E_{0}^{\prime}=E_{0}$.

In the second order of the expansion in $\beta$ we have

$$
\begin{aligned}
i \partial_{t} c_{n}^{(2)} & =-E_{n}^{(1)} c_{n}^{(1)}-E_{n}^{(2)} c_{n}^{(0)}+ \\
& +\sum_{m_{1} m_{2} m_{3}} V_{n}^{m_{1} m_{2} m_{3}}\left(c_{m_{1}}^{*(1)} c_{m_{2}}^{(0)} c_{m_{3}}^{(0)}+2 c_{m_{1}}^{*(0)} c_{m_{2}}^{(1)} c_{m_{3}}^{(0)}\right) e^{i\left(E_{n}^{\prime}+E_{m_{1}}^{\prime}-E_{m_{2}}^{\prime}-E_{m_{3}}^{\prime}\right) t} \\
& =-E_{n}^{(2)} \delta_{n 0}-E_{n}^{(1)} c_{n}^{(1)}+\sum_{m_{1}} V_{n}^{m_{1} 00}\left(c_{m_{1}}^{*(1)} e^{i\left(E_{n}^{\prime}+E_{m_{1}}^{\prime}-2 E_{0}^{\prime}\right) t}+2 c_{m_{1}}^{(1)} e^{i\left(E_{n}^{\prime}-E_{m_{1}}^{\prime}\right) t}\right) .
\end{aligned}
$$

For $n=0$ it takes the form

$$
i \partial_{t} c_{0}^{(2)}=-E_{0}^{(2)}+\sum_{m} V_{0}^{m 00}\left(c_{m}^{*(1)} e^{i\left(E_{m}^{\prime}-E_{0}^{\prime}\right) t}+2 c_{m}^{(1)} e^{i\left(E_{0}^{\prime}-E_{m}^{\prime}\right) t}\right) .
$$

Substitution of (3.9) and (3.12) yields

$$
\begin{aligned}
i \partial_{t} c_{0}^{(2)} & =-E_{0}^{(2)}+\sum_{m \neq 0} \frac{V_{0}^{m 00} V_{m}^{000}}{E_{m}^{\prime}-E_{0}^{\prime}}\left[\left(1-e^{-i\left(E_{m}^{\prime}-E_{0}^{\prime}\right) t}\right) e^{i\left(E_{m}^{\prime}-E_{0}^{\prime}\right) t}+\right. \\
& \left.+2\left(1-e^{i\left(E_{m}^{\prime}-E_{0}^{\prime}\right) t}\right) e^{i\left(E_{0}^{\prime}-E_{m}^{\prime}\right) t}\right] \\
& =-E_{0}^{(2)}+\sum_{m \neq 0} \frac{V_{0}^{m 00} V_{m}^{000}}{E_{m}^{\prime}-E_{0}^{\prime}}\left(e^{i\left(E_{m}^{\prime}-E_{0}^{\prime}\right) t}+2 e^{i\left(E_{0}^{\prime}-E_{m}^{\prime}\right) t}-3\right),
\end{aligned}
$$


and the secular term could be removed by setting

$$
E_{0}^{(2)}=-3 \sum_{m \neq 0} \frac{V_{0}^{m 00} V_{m}^{000}}{E_{m}^{\prime}-E_{0}^{\prime}}
$$

For $n \neq 0$ we have

$$
\begin{aligned}
i \partial_{t} c_{n}^{(2)} & =-E_{n}^{(1)} V_{n}^{000}\left(\frac{1-e^{i\left(E_{n}^{\prime}-E_{0}^{\prime}\right) t}}{E_{n}^{\prime}-E_{0}^{\prime}}\right)+ \\
& +\sum_{m} V_{n}^{m 00}\left(c_{m}^{*(1)} e^{i\left(E_{n}^{\prime}+E_{m}^{\prime}-2 E_{0}^{\prime}\right) t}+2 c_{m}^{(1)} e^{i\left(E_{n}^{\prime}-E_{m}^{\prime}\right) t}\right) \\
= & -E_{n}^{(1)} V_{n}^{000}\left(\frac{1-e^{i\left(E_{n}^{\prime}-E_{0}^{\prime}\right) t}}{E_{n}^{\prime}-E_{0}^{\prime}}\right)+ \\
& +\sum_{m \neq 0} \frac{V_{n}^{m 00} V_{m}^{000}}{E_{m}^{\prime}-E_{0}^{\prime}}\left(e^{i\left(E_{n}^{\prime}+E_{m}^{\prime}-2 E_{0}^{\prime}\right) t}+2 e^{i\left(E_{n}^{\prime}-E_{m}^{\prime}\right) t}-3 e^{i\left(E_{n}^{\prime}-E_{0}^{\prime}\right) t}\right)
\end{aligned}
$$

We notice that the second term in the sum produces secular terms for $m=n$. Those terms could be removed by setting

$$
\begin{gathered}
-\frac{E_{n}^{(1)} V_{n}^{000}}{E_{n}^{\prime}-E_{0}^{\prime}}+\frac{2 V_{n}^{n 00} V_{n}^{000}}{E_{n}^{\prime}-E_{0}^{\prime}}=0 \quad n \neq 0 \\
E_{n}^{(1)}=2 V_{n}^{n 00} \quad n \neq 0 .
\end{gathered}
$$

To conclude, up to the second order in $\beta$, the perturbed energies, which are required to remove the secular terms, are given by

$$
E_{n}^{\prime}=E_{n}^{(0)}+\beta V_{n}^{n 00}\left(2-\delta_{n 0}\right)-3 \beta^{2} \delta_{n 0} \sum_{m \neq 0} \frac{\left(V_{m}^{000}\right)^{2}}{E_{m}^{\prime}-E_{0}^{\prime}}
$$

and the corresponding correction to $c_{n}^{(0)}$ is

$$
i \partial_{t} c_{n}^{(2)}=\left\{\begin{array}{cc}
\sum_{m \neq 0} \frac{V_{0}^{m 00} V_{m}^{000}}{E_{m}^{\prime}-E_{0}^{\prime}}\left(e^{i\left(E_{m}^{\prime}-E_{0}^{\prime}\right) t}+2 e^{i\left(E_{0}^{\prime}-E_{m}^{\prime}\right) t}\right) & n=0 \\
\frac{2 V_{n}^{n 00} V_{n}^{000}}{E_{n}^{\prime}-E_{0}^{\prime}} e^{i\left(E_{n}^{\prime}-E_{0}^{\prime}\right) t}+\sum_{m \neq 0, n} \frac{2 V_{n}^{m 00} V_{m}^{000}}{E_{m}^{\prime}-E_{0}^{\prime}} e^{i\left(E_{n}^{\prime}-E_{m}^{\prime}\right) t}+ & n \neq 0 \\
+\sum_{m \neq 0} \frac{V_{n}^{m 00} V_{m}^{000}}{E_{m}^{\prime}-E_{0}^{\prime}}\left(e^{i\left(E_{n}^{\prime}+E_{m}^{\prime}-2 E_{0}^{\prime}\right) t}-3 e^{i\left(E_{n}^{\prime}-E_{0}^{\prime}\right) t}\right) &
\end{array} .\right.
$$

Note that in the calculation of $c_{n}$ to higher orders in $\beta$, a secular term of the order $\beta^{2}$ will be generated for $n \neq 0$. Secular terms with increasing complexity are generated in the cancellation of higher orders, however, as demonstrated by (3.7), secular terms are removed with the same $c_{n}^{(0)}$ and $c_{n}^{(1)}$ which are presented in $(2.15,3.12)$.

In the next section, the entropy problem will be studied. It will be shown that the proliferation of terms in the expansion is at most exponential. 


\section{The ENTRopy PRoBlem}

Since the time dependence of all orders is bounded (excluding the secular terms), we can bound each order of the expansion by

$$
\begin{aligned}
\left|c_{n}^{(0)}\right| & =\delta_{n 0} \\
\left|c_{n}^{(1)}\right| & =\left|V_{n}^{000}\left(\frac{1-e^{i\left(E_{n}^{\prime}-E_{0}^{\prime}\right) t}}{E_{n}^{\prime}-E_{0}^{\prime}}\right)\right| \leq 2\left|\frac{V_{n}^{000}}{E_{n}^{\prime}-E_{0}^{\prime}}\right| \\
\left|c_{n}^{(2)}\right| & =\left|\sum_{m} \int_{0}^{t} d t^{\prime}\left(\frac{V_{n}^{m 00} V_{m}^{000}}{E_{m}^{\prime}-E_{0}^{\prime}}\right)\left(e^{i\left(E_{n}^{\prime}+E_{m}^{\prime}-2 E_{0}^{\prime}\right) t^{\prime}}-3 e^{i\left(E_{n}^{\prime}-E_{0}^{\prime}\right) t^{\prime}}+2 e^{i\left(E_{n}^{\prime}-E_{m}^{\prime}\right) t^{\prime}}\right)\right| \\
& \leq 2 \cdot \sum_{m} \frac{\left|V_{n}^{m 00}\right|\left|V_{m}^{000}\right|}{\left|E_{m}^{\prime}-E_{0}^{\prime}\right|}\left(\frac{1}{\left|\left(E_{n}^{\prime}+E_{m}^{\prime}-2 E_{0}^{\prime}\right)\right|}+\frac{3}{\left|E_{n}^{\prime}-E_{0}^{\prime}\right|}+\frac{2}{\left|E_{n}^{\prime}-E_{m}^{\prime}\right|}\right)
\end{aligned}
$$

et cetera. However, for convergence for a finite but possibly small $\beta$, it is essential that the number of terms on the RHS of (4.1) will not increase faster than exponentially in $k$, e.g. not as $k$ !, where $k$ is the expansion order. Next we will show that the number of terms indeed increases at most exponentially in $k$.

We will designate the number of different products of order $k$ of $V^{\prime} s$ by $R_{k}$ (on top of it there is still a number of non vanishing terms in the sums over $m$, that will be estimated in the next section). By replacing each $c_{n}^{(l)}$ in (3.6) by $R_{l}$ (the integration with respect to time multiples the number of terms by a factor of 2 , cf. (4.1)) we deduce a recursive expression for $R_{k}$

$$
R_{k}=\sum_{r=0}^{k-1} \sum_{l=0}^{k-1-r} R_{r} R_{l} R_{k-1-r-l} \quad R_{1}=1 \quad R_{0}=1 .
$$

In order to find an upper bound on $R_{k}$ we examine the structure of the products of $V^{\prime} s$ we notice that each product could be uniquely labeled by a vector of zeros and $m_{i}^{\prime} s$

$$
V_{n}^{m_{1} 00} V_{m_{1}}^{m_{2} m_{3} 0} \cdots V_{m_{k-2}}^{0 m_{k-1} 0} V_{m_{k-1}}^{000} \rightarrow\left\{m_{1}, 0,0, m_{2}, m_{3}, 0, \cdots, 0, m_{k-1}, 0,0,0,0\right\},
$$

where the number of different summation indices $m_{i}$ is $(k-1)$ and the length of the labeling vector is $3 k$. Since in each vector the last three elements should always be zeros the number of different configurations of this product is the number of ways to distribute $(k-1) m^{\prime} s$ in $3(k-1)$ cells (superscripts), namely, $R_{k}<\left(\begin{array}{c}3(k-1) \\ k-1\end{array}\right)$. This is only an upper bound, since there may be some additional constraints, for example, the first three elements in the vector should never be all zeros. Subtracting the cases when all three first elements are zero $\left(\begin{array}{c}3(k-2) \\ k-1\end{array}\right)$ we obtain the bound

$$
R_{k}<\left(\begin{array}{c}
3(k-1) \\
k-1
\end{array}\right)-\left(\begin{array}{c}
3(k-2) \\
k-1
\end{array}\right)
$$

This bound has the following asymptotics

$$
\lim _{k \rightarrow \infty} \frac{1}{k} \ln \left[\left(\begin{array}{c}
3 k-3 \\
k-1
\end{array}\right)-\left(\begin{array}{c}
3 k-6 \\
k-1
\end{array}\right)\right]=\ln \frac{27}{4},
$$


namely,

$$
R_{k} \leq e^{k \ln \frac{27}{4}} \leq e^{2 k}
$$

From Fig.4.1 we conclude that this bound is a tight bound of the exact solution of the recurrence relation. This bound shows that the number of terms in the

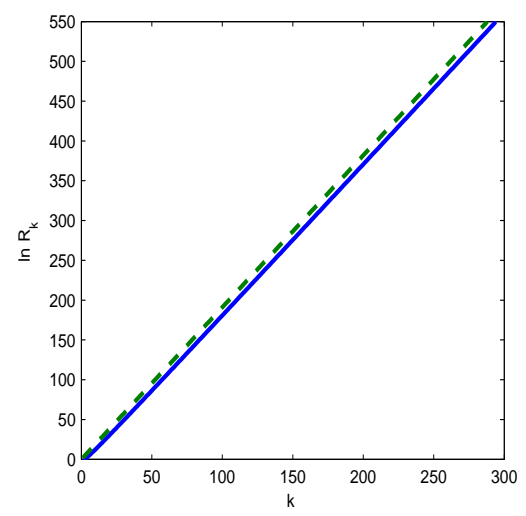

Figure 4.1. The solid line denotes the exact numerical solution of the recurrence relation for $R_{k}$ and dashed line is the asymptotic upper bound on this solution $\left(e^{2 k}\right)$.

expansion increases at most exponentially in $k$ and therefore there is no entropy problem.

\section{Bounding THE General TERM}

As clear from (3.6) after the subtraction of all the secular terms in the preceding orders the differential equation for the $k$-th order term is

$$
\begin{aligned}
i \partial_{t} c_{n}^{(k)} & =-\sum_{s=0}^{k-1} E_{n}^{(k-s)} c_{n}^{(s)}+ \\
& +\sum_{m_{1} m_{2} m_{3}} V_{n}^{m_{1} m_{2} m_{3}}\left[\sum_{r=0}^{k-1} \sum_{s=0}^{k-1-r} \sum_{l=0}^{k-1-r-s} c_{m_{1}}^{(r) *} c_{m_{2}}^{(s)} c_{m_{3}}^{(l)}\right] e^{i\left(E_{n}^{\prime}+E_{m_{1}}^{\prime}-E_{m_{2}}^{\prime}-E_{m_{3}}^{\prime}\right) t} .
\end{aligned}
$$

with the initial condition of $c_{n}^{(0)}=\delta_{n 0}$ and the first term on the RHS is designed (see Section 3) to eliminate all the time-independent part of of RHS of (5.1). Following the construction of the lower order terms in the preceding section by a repeated application of (5.1) the structure of the general term in the expression for a given order $k$ can be obtained. Note that the structure $E_{n}^{(l)}$ is similar to the structure of $c_{n}^{(l)}$. The main blocks of the structure take the form

$$
\zeta_{n}^{m_{1} m_{2} m_{3}} \equiv \frac{V_{n}^{m_{1} m_{2} m_{3}}}{E_{n}^{\prime}-\left\{E^{\prime}\right\}_{m_{i}}}
$$

where $\left\{E^{\prime}\right\}_{m_{i}}$ denotes a sum of eigenenergies (shifted so that the secular terms are removed, see (3.2)) that may depend on the summation indices $m_{i}$. Then any term 
of order $k$ is a product of $k$ factors of the form (5.2) and $k-1$ summations over the indices $m_{i}$

$$
\overbrace{\zeta_{n}^{m_{1} m_{2} m_{3}} \zeta_{m_{1}}^{m_{4} m_{5} m_{6}} \cdots \zeta_{m_{5}}^{000} \zeta_{m_{6}}^{000}}^{k \text { terms }}
$$

and following the last section there is an exponentially increasing (in $k$ ) number of such terms. In order to bound the general term of order $k$ we will first bound one typical block, namely,

$$
\zeta_{n}^{m_{1} m_{2} m_{3}} \equiv \frac{V_{n}^{m_{1} m_{2} m_{3}}}{E_{n}^{\prime}-\left\{E^{\prime}\right\}_{m_{i}}}
$$

where $\left\{E^{\prime}\right\}_{m_{i}}$ is some sum of $E_{j}^{\prime}$. To bound (5.4) we will bound separately the denominator and the numerator. Using Cauchy-Schwarz inequality,

$$
\left\langle\left|\zeta_{n}^{m_{1} m_{2} m_{3}}\right|^{s}\right\rangle \leq\left\langle\frac{1}{\left|E_{n}^{\prime}-\left\{E^{\prime}\right\}_{m_{i}}\right|^{2 s}}\right\rangle^{1 / 2}\left\langle\left|V_{n}^{m_{1} m_{2} m_{3}}\right|^{2 s}\right\rangle^{1 / 2},
$$

where $0<s<\frac{1}{2}$.

Conjecture 3. For the Anderson model, which is given by the linear part of (1.1), the joint distribution of $R$ eigenenergies is bounded,

$$
p\left(E_{1}, E_{2}, \ldots, E_{R}\right) \leq \bar{D}_{R}
$$

where $\bar{D}_{R} \propto R !<\infty$.

The conjecture is inspired by Theorem (3.1) of the recent paper by Aizenman and Warzel [62]. If one assumes that with probability one the profiles of the eigenfunctions, namely, the squares of the eigenfunctions, which correspond to the eigenenergies $\left\{E_{i}\right\}_{i=1}^{R}$ are substantially different such that $\alpha$ (as defined in Theorem (3.1) of [62]) is bounded away from zero, than taking the intervals $I_{j}=d E_{j}$ one finds that the joint probability density can be bounded by $\bar{D}_{R} \propto \frac{R}{\alpha^{R}}$. It is not known how to prove that for the Anderson model the profiles of the wave functions are distnict and how to quantify this. However, it is reasonable to assume distinctness since different eigenfunctions are localized in different regions and therefore are affected by different potentials. There are double humped states (consisting of nearly symmetric and antisymmetric combinations of two humps), which have approximately the same squares, and therefore are natural candidates for states that may result in violation of the conjecture. Nevertheless, those states are very rare and the difference between their squares is exponential in the distance between the humps. For this it is crucial that many sites are invloved (therefore the counter example (2.1) of [62] is not generic). If the energies are assigned to specific locations than the factorial term could be dropped, namely, $\bar{D}_{R} \propto \alpha^{-R}$. This is due to the fact that specific assignment of energies chooses one of the $R$ ! permutations, mentioned in [62].

Corollary 4. Given $0<s<1$, for $f=\sum_{k=1}^{R} c_{k} E_{i_{k}}$, where $c_{k}$ are integers (and the assignment of eigenfunctions to sites is given by Definition 1) the following mean is bounded from above

$$
\left\langle\frac{1}{|f|^{s}}\right\rangle \leq D_{R}<\infty
$$


where $D_{R} \propto \bar{D}_{R}$.

Proof. By Conjecture 3,

$$
\left\langle\frac{1}{|f|^{s}}\right\rangle=\int \frac{p\left(E_{1}, E_{2}, \ldots, E_{R}\right) d E_{1} d E_{2} \cdots d E_{R}}{\left|\sum_{k=1}^{R} c_{k} E_{i_{k}}\right|^{s}} \leq \bar{D}_{R} \int \frac{d E_{1} d E_{2} \cdots d E_{R}}{\left|\sum_{k=1}^{R} c_{k} E_{i_{k}}\right|^{s}},
$$

changing the variables to $\left\{f, E_{2}, E_{3}, \ldots, E_{R}\right\}$ gives

$$
\left\langle\frac{1}{|f|^{s}}\right\rangle \leq \bar{D}_{R} \int_{-\Delta}^{\Delta} \frac{d E_{2} \cdots d E_{R}}{\left|c_{1}\right|} \int_{f(\vec{E})} d f \frac{1}{|f|^{s}},
$$

where $\left|c_{1}\right|$ is the Jacobian and $2 \Delta$ is the support of the energies. Due to the fact that $f\left(E_{1}\right)$ is linear the multiplicity is one. Since the integrand is positive we can only increase the integral by increasing the domain of integration of $f$. Designating by $f_{\infty}$ the maximal value of $f$,

$$
\left\langle\frac{1}{|f|^{s}}\right\rangle \leq 2 \bar{D}_{R} \Delta^{R-1} \frac{f_{\infty}^{1-s}}{1-s} \equiv D_{R}
$$

Conjecture 5. In the limit of $R \rightarrow \infty$, for $0<s<1$ and for $f=\sum_{k=1}^{R} c_{k} E_{i_{k}}$, where $c_{k}$ are integers (and the assignment of eigenfunctions to sites is given by Definition 1)

$$
\left\langle\frac{1}{|f|^{s}}\right\rangle \asymp \frac{1}{R^{s / 2}}
$$

For large $R$ the sum, $f=\sum_{k=1}^{R} c_{k} E_{i_{k}}$, can be effectively separated into groups of terms that depend on different diagonal energies, $\varepsilon_{j}$. Therefore by the central limit theorem, $f$ is effectively a Gaussian variable with $\langle f\rangle=0$ and $\left\langle f^{2}\right\rangle=\sigma^{2} R$, where $\sigma^{2}$ is some constant. Therefore,

$$
\left\langle\frac{1}{|f|^{s}}\right\rangle \asymp \frac{2}{\sqrt{2 \pi \sigma^{2} R}} \int_{0}^{\infty} \frac{d f}{f^{s}} e^{-f^{2} /\left(2 \sigma^{2} R\right)}=\frac{2}{\sqrt{2 \pi}\left(\sqrt{\sigma^{2} R}\right)^{s}} \int_{0}^{\infty} \frac{d f}{f^{s}} e^{-f^{2} / 2} \asymp R^{-\frac{s}{2}} .
$$

Conjecture 3 and Corollary 4 were tested numerically for lattice size 128, $s=\frac{1}{2}$ and the uniform distribution

$$
\mu(\varepsilon(x))= \begin{cases}\frac{1}{2 \Delta} & |\varepsilon(x)| \leq \Delta \\ 0 & |\varepsilon(x)|>\Delta\end{cases}
$$

with $\Delta=1$. The results are presented in Fig. 5.1 for $R \leq 10$. For $R \leq 3$ all the combinations of the energies were used and the result is an average over all the combinations. For $R \geq 4$ only a partial set of combinations of cardinality $10^{4}$, chosen at random was used. For large $R$ the decay is as $R^{-s / 2}$ in agreement with Conjecture 5. The above calculation was repeated for the case where the $E_{i}$ are replaced by the renormalized energies $E_{i}^{\prime}$. The calculation can be performed only to the order $\beta^{2}$ with the help of (3.18). The results are also presented in Fig. 5.1 for $\beta=0.1$ and $\beta=1$. 


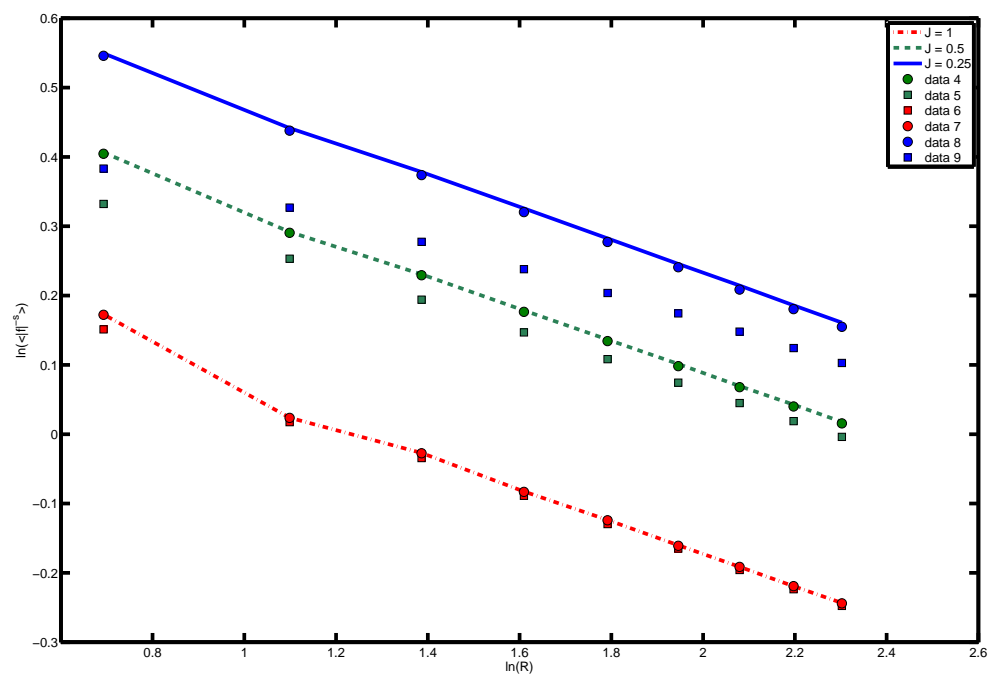

Figure 5.1. The logarithm of $\left\langle|f|^{-1 / 2}\right\rangle$ as a function of the logarithm of $R$. The lines designate denominators with $\beta=0$, with the solid line (blue) is for $J=0.25$ the dashed line (green) is for $J=0.5$ and the dot-dashed line (red) is for $J=1$. The solid circles and the squares are data with $\beta=1$, and $E_{n}^{\prime}$ calculated up to the second in $\beta$, such that different colors represent different $J$, in the similar manner as for the lines. The solid squares are for parameters similar to the ones with the solid circles, but with the restriction that at least one of the states that corresponds to $E_{n}^{\prime}$ which is localized near the origin.

Conjecture 6. Corollary 4 and Conjecture 5 hold also if the $E_{i}$ are replaced by the renormalized energies $E_{i}^{\prime}$.

The reason is that the various renormalized energies are dominated by different independent random variables $\varepsilon_{i}$. The numerical calculations support this point of view. In what follows Corollary 4 and Conjecture 6 (and not Conjecture 3) are used, and these were tested numerically (Fig. 5.1).

Using the bound on the overlap sum (2.11) and Corollary 4,

$$
\left\langle\left|\zeta_{n}^{m_{1} m_{2} m_{3}}\right|^{s}\right\rangle_{\delta} \leq D_{\delta}\left|V_{\delta}^{\varepsilon, \varepsilon^{\prime}}\right|^{s} e^{\varepsilon s\left(\left|x_{n}\right|+\left|x_{m_{1}}\right|+\left|x_{m_{2}}\right|+\left|x_{m_{3}}\right|\right)} e^{-\frac{1}{3}\left(\gamma-\varepsilon^{\prime}\right) s\left(\left|x_{n}-x_{m_{1}}\right|+\left|x_{n}-x_{m_{2}}\right|+\left|x_{n}-x_{m_{3}}\right|\right)} .
$$

this proves the proposition:

Proposition 7. For some $\delta, \varepsilon, \varepsilon^{\prime}>0$ and $0<s<\frac{1}{2}$,

$$
\left\langle\left|\zeta_{n}^{m_{1} m_{2} m_{3}}\right|^{s}\right\rangle_{\delta} \leq F^{\prime} e^{\varepsilon s\left(\left|x_{n}\right|+\sum_{i}\left|x_{m_{i}}\right|\right)} e^{-\frac{1}{3}\left(\gamma-\varepsilon^{\prime}\right) s \sum_{i}\left|x_{n}-x_{m_{i}}\right|}
$$

where $F^{\prime}=D_{\delta}\left|V_{\delta}^{\varepsilon, \varepsilon^{\prime}}\right|^{s}$. 
Using the Chebyshev inequality,

$$
\operatorname{Pr}(|x| \geq a) \leq\langle|x|\rangle / a,
$$

where $x$ is a random variable and $a$ is a constant, one finds

\section{Corollary 8.}

(5.16)

$\operatorname{Pr}\left(\left|\zeta_{n}^{m_{1} m_{2} m_{3}}\right| \geq F^{\prime 1 / s} e^{\varepsilon\left(\left|x_{n}\right|+\sum_{i}\left|x_{m_{i}}\right|\right)} e^{-\frac{1}{3}\left(\gamma-\varepsilon^{\prime}-\eta\right) \sum_{i}\left|x_{n}-x_{m_{i}}\right|}\right) \leq e^{-\frac{\eta s}{3} \sum_{i}\left|x_{n}-x_{m_{i}}\right|}$

where $F^{\prime}=D_{\delta}\left|V_{\delta}^{\varepsilon, \varepsilon^{\prime}}\right|^{s}$.

A general term in the expression for different orders of $c_{n}^{(k)}$ is given by the form of $\left|\zeta_{n}^{m_{1} m_{2} m_{3}}\right|\left|\zeta_{m_{1}}^{m_{4} m_{5} m_{6}}\right| \cdots\left|\zeta_{m_{k-1}}^{000}\right|$, i.e., it contains $(k-1)$ summations over indices which run over all the lattice. First we construct a general procedure to bound a product of $k, \zeta$ 's. A product of two $\zeta$ 's is bounded by

$$
\left\langle\left|\sum_{m_{1}} \zeta_{n}^{m_{1} m_{2} m_{3}} \zeta_{m_{1}}^{m_{4} m_{5} m_{6}}\right|^{s}\right\rangle_{\delta} \leq\left\langle\sum_{m_{1}}\left|\zeta_{n}^{m_{1} m_{2} m_{3}}\right|^{s}\left|\zeta_{m_{1}}^{m_{4} m_{5} m_{6}}\right|^{s}\right\rangle_{\delta},
$$

where $\langle\cdot\rangle_{\delta}$ denotes an average over realizations where (2.4) is satisfied.

Using the Cauchy-Shwarz inequality

$$
\sum_{m_{1}}\left\langle\left|\zeta_{n}^{m_{1} m_{2} m_{3}}\right|^{s}\left|\zeta_{m_{1}}^{m_{4} m_{5} m_{6}}\right|^{s}\right\rangle_{\delta} \leq \sum_{m_{1}}\left\langle\left|\zeta_{n}^{m_{1} m_{2} m_{3}}\right|^{2 s}\right\rangle_{\delta}^{1 / 2}\left\langle\left|\zeta_{m_{1}}^{m_{4} m_{5} m_{6}}\right|^{2 s}\right\rangle_{\delta}^{1 / 2}
$$

setting $0<s<\frac{1}{4}$ (notice, that $s<\frac{1}{4}$ and not $s<\frac{1}{2}$, due to (5.5)) and inserting

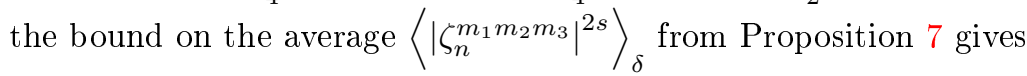

$$
\begin{aligned}
& \left\langle\left|\sum_{m_{1}} \zeta_{n}^{m_{1} m_{2} m_{3}} \zeta_{m_{1}}^{m_{4} m_{5} m_{6}}\right|^{s}\right\rangle_{\delta} \\
& \leq F^{\prime} \exp \left[\varepsilon s\left(\left|x_{n}\right|+\sum_{i=2}^{6}\left|x_{m_{i}}\right|\right)\right] e^{-s \frac{\gamma-\varepsilon^{\prime}}{3}\left(\left|x_{n}-x_{m_{2}}\right|+\left|x_{n}-x_{m_{3}}\right|\right)} \times \\
& \times \sum_{m_{1}} e^{2 \varepsilon s\left|x_{m_{1}}\right|} \exp -s \frac{\gamma-\varepsilon^{\prime}}{3}\left(\left|x_{n}-x_{m_{1}}\right|+\sum_{i=4}^{6}\left|x_{m_{1}}-x_{m_{i}}\right|\right)
\end{aligned}
$$

where we have used the inequality

$$
\left(\sum_{i}\left|x_{i}\right|\right)^{s} \leq \sum_{i}\left|x_{i}\right|^{s} \quad 0<s<1 .
$$

Using the triangle inequality in the same manner as in (2.12)

$$
\left|x_{n}-x_{m_{1}}\right|+\sum_{i=4}^{6}\left|x_{m_{1}}-x_{m_{i}}\right| \geq \frac{1}{3} \sum_{i=4}^{6}\left|x_{n}-x_{m_{i}}\right|+\frac{2}{3} \sum_{i=4}^{6}\left|x_{m_{1}}-x_{m_{i}}\right|
$$


we get

$$
\begin{aligned}
& \left\langle\left|\sum_{m_{1}} \zeta_{n}^{m_{1} m_{2} m_{3}} \zeta_{m_{1}}^{m_{4} m_{5} m_{6} \mid}\right|_{\delta}^{s}\right\rangle_{i=2} F^{\prime} \exp \varepsilon s\left(\left|x_{n}\right|+\sum_{i=2}^{6}\left|x_{m_{i}}\right|\right) e^{-\frac{\gamma-\varepsilon^{\prime}}{3} s\left(\left|x_{n}-x_{m_{2}}\right|+\left|x_{n}-x_{m_{3}}\right|\right)_{\times}} \\
& \times \exp \left[-\frac{\gamma-\varepsilon^{\prime}}{9} s \sum_{i=4}^{6}\left|x_{n}-x_{m_{i}}\right|\right] \sum_{m_{1}} e^{2 \varepsilon s\left|x_{m_{1}}\right|} e^{-2 \frac{\gamma-\varepsilon^{\prime}}{3} s \sum_{i=4}^{6}\left|x_{m_{1}}-x_{m_{i}}\right|} \\
& =F^{\prime \prime} \exp \left[s \varepsilon\left(\left|x_{n}\right|+\sum_{i=2}^{6}\left|x_{m_{i}}\right|\right)-\frac{\gamma-\varepsilon^{\prime}}{9} s \sum_{i=4}^{6}\left|x_{n}-x_{m_{i}}\right|\right] e^{-\frac{\gamma-\varepsilon^{\prime}}{3} s\left(\left|x_{n}-x_{m_{2}}\right|+\left|x_{n}-x_{m_{3}}\right|\right),}
\end{aligned}
$$

where $F^{\prime \prime}\left(\gamma, \varepsilon^{\prime}, s, \varepsilon\right)=F^{\prime} \sum_{m_{1}} e^{2 \varepsilon s\left|x_{m_{1}}\right|} e^{-2 \frac{\gamma-\varepsilon^{\prime}}{3} s \sum_{i=4}^{6}\left|x_{m_{1}}-x_{m_{i}}\right|}<\infty$, in the following also other convergent sums of this type will be denoted by $F^{\prime \prime}$.

If the term we consider is a term in the perturbation expansion it should include some factors $\zeta_{m}^{m_{1} m_{2} m_{3}}$ with some $m_{i}=0$. A simple example is where $x_{m_{1}}=x_{m_{2}}=x_{m_{3}}=0$

$$
\left\langle\left|\zeta_{m}^{000}\right|^{s}\right\rangle_{\delta} \leq F^{\prime} e^{-s\left(\gamma-\varepsilon^{\prime}-\varepsilon\right)\left|x_{n}\right|}
$$

where the bound was calculated using Proposition 7. The product should terminate with a term of the form $\zeta_{m}^{000}$ therefore a term like (5.17) is a part of a product of the form,

$$
\sum_{\left\{m_{i}\right\}}\left|\zeta_{n}^{m_{1} m_{2} m_{3}}\right|\left|\zeta_{m_{1}}^{m_{4} m_{5} m_{6}}\right|\left|\zeta_{m_{2}}^{000}\right|\left|\zeta_{m_{3}}^{000}\right|\left|\zeta_{m_{4}}^{000}\right|\left|\zeta_{m_{5}}^{000}\right|\left|\zeta_{m_{6}}^{000}\right|
$$

To bound it we use the generalized Hölder inequality,

$$
\left\langle\prod_{i=1}^{k}\left|x_{i}\right|\right\rangle \leq \prod_{i=1}^{k}\left\langle\left|x_{i}\right|^{k}\right\rangle^{1 / k}
$$

Applying it yields,

$$
\begin{aligned}
& \sum_{\left\{m_{i}\right\}}\left\langle\left|\zeta_{n}^{m_{1} m_{2} m_{3}}\right|^{s}\left|\zeta_{m_{1}}^{m_{4} m_{5} m_{6}}\right|^{s}\left|\zeta_{m_{2}}^{000}\right|^{s}\left|\zeta_{m_{3}}^{000}\right|^{s}\left|\zeta_{m_{4}}^{000}\right|^{s}\left|\zeta_{m_{5}}^{000}\right|^{s}\left|\zeta_{m_{6}}^{000}\right|^{s}\right\rangle_{\delta} \\
& \leq \sum_{\left\{m_{i}\right\}}\left(\left\langle\left|\zeta_{n}^{m_{1} m_{2} m_{3}}\right|^{7 s}\right\rangle_{\delta}\left\langle\left|\zeta_{m_{1}}^{m_{4} m_{5} m_{6}}\right|^{7 s}\right\rangle_{\delta} \prod_{i=2}^{6}\left\langle\left|\zeta_{m_{i}}^{000}\right|^{7 s}\right\rangle_{\delta}\right)^{1 / 7}
\end{aligned}
$$




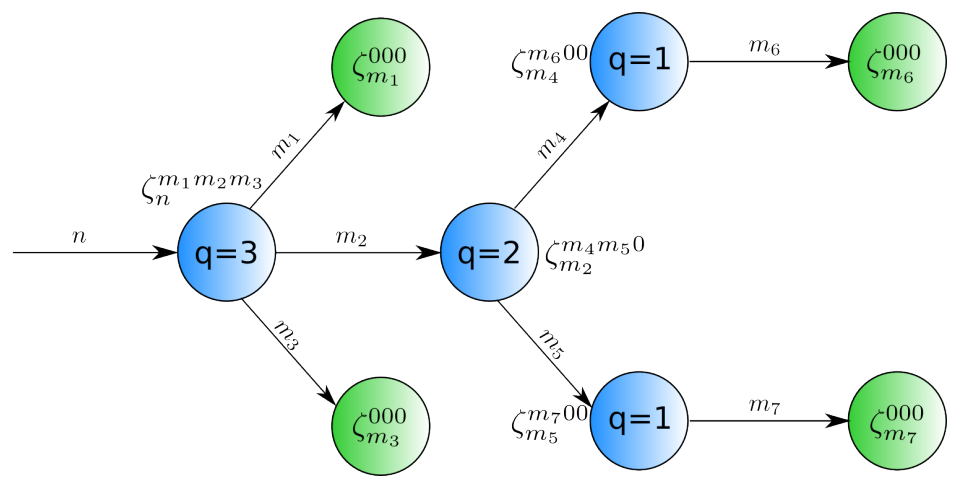

Figure 5.2. An example of a graph that is used to construct the general term. The graph describes an 8-th order term, $\zeta_{n}^{m_{1} m_{2} m_{3}} \zeta_{m_{1}}^{000} \zeta_{m_{3}}^{000} \zeta_{m_{2}}^{m_{4} m_{5} 0} \zeta_{m_{4}}^{m_{6} 00} \zeta_{m_{5}}^{m_{7} 00} \zeta_{m_{6}}^{000} \zeta_{m_{7}}^{000}$

setting $0<s<\frac{1}{14}$ and inserting the bounds on the averages (5.17) and (5.20) gives

$$
\begin{aligned}
& \left\langle\left(\sum_{\left\{m_{i}\right\}} \zeta_{n}^{m_{1} m_{2} m_{3}} \zeta_{m_{1}}^{m_{4} m_{5} m_{6}} \zeta_{m_{2}}^{000} \zeta_{m_{3}}^{000} \zeta_{m_{4}}^{000} \zeta_{m_{5}}^{000} \zeta_{m_{6}}^{000}\right)^{s}\right\rangle_{\delta} \\
& \leq F^{\prime} \sum_{\left\{m_{i}\right\}} \exp \varepsilon s\left(\left|x_{n}\right|+\sum_{i=2}^{6}\left|x_{m_{i}}\right|\right) e^{-\frac{\gamma-\varepsilon^{\prime}}{3} s\left(\left|x_{n}-x_{m_{2}}\right|+\left|x_{n}-x_{m_{3}}\right|\right)_{\times}} \\
& \times \exp \left[-\frac{\gamma-\varepsilon^{\prime}}{9} s \sum_{i=4}^{6}\left|x_{n}-x_{m_{i}}\right|-\left(\gamma-\varepsilon^{\prime}-\varepsilon\right) s \sum_{i=2}^{6}\left|x_{m_{i}}\right|\right] \\
& =F^{\prime} e^{\varepsilon s\left|x_{n}\right|} \sum_{\left\{m_{i}\right\}} e^{-\frac{\gamma-\varepsilon^{\prime}}{3}} s\left(\left|x_{n}-x_{m_{2}}\right|+\left|x_{n}-x_{m_{3}}\right|\right) \times \\
& \times \exp \left[-\frac{\gamma-\varepsilon^{\prime}}{9} s \sum_{i=4}^{6}\left|x_{n}-x_{m_{i}}\right|-\left(\gamma-\varepsilon^{\prime}-2 \varepsilon\right) s \sum_{i=2}^{6}\left|x_{m_{i}}\right|\right]
\end{aligned}
$$

where $\left\{m_{i}\right\}$ stands for a sum over all the $m_{i}$. Using the inequality,

$$
\sum_{i=4}^{6}\left(\left|x_{n}-x_{m_{i}}\right|+\left|x_{m_{i}}\right|\right) \geq 3\left|x_{n}\right|
$$

we get

$$
\begin{aligned}
& \left\langle\left(\sum_{\left\{m_{i}\right\}} \zeta_{n}^{m_{1} m_{2} m_{3}} \zeta_{m_{1}}^{m_{4} m_{5} m_{6}} \zeta_{m_{2}}^{000} \zeta_{m_{3}}^{000} \zeta_{m_{4}}^{000} \zeta_{m_{5}}^{000} \zeta_{m_{6}}^{000}\right)^{s}\right\rangle_{\delta} \\
& \leq F^{\prime} e^{-\left(\gamma-\varepsilon^{\prime}-\varepsilon\right) s\left|x_{n}\right|} \sum_{\left\{m_{i}\right\}} \exp -2 s\left(\frac{\gamma-\varepsilon^{\prime}}{3}-\varepsilon\right) \sum_{i=2}^{6}\left|x_{m_{i}}\right| \\
& =F^{\prime} e^{-\left(\gamma-\varepsilon^{\prime}-\varepsilon\right) s\left|x_{n}\right|} .
\end{aligned}
$$

Let us study the form of a general graph (c.f. Fig. 5.2). 
It can be described as a tree starting from $n$, the "root" and four types of branching points, where $q(0 \leq q \leq 3)$ branches continue while $3-q$ branches terminate. A branch which continues is associated with a value $m_{i} \neq 0$, while a branch terminates with $m_{i}=0$. In the above, bounds on branches with $q=0$ and 3 are calculated, and are given by (5.14) and (5.20), respectively. The bounds for $q=1,2$ follow similarly from Proposition 7. Along each "bond" from the "root" to the "leaves" a term $\zeta_{n}^{m_{1} m_{2} m_{3}}$ is multiplied. At a point from where $q$ branches continue the exponent is reduced by a factor of $q$. In other words, with $x_{n}$ and $x_{m}$ are connected by a path that is crossing $l$ branching points with ratios $q_{1}, \ldots, q_{l}$ the bound on the product of the zetas contains a factor $\exp -\frac{\gamma}{\tilde{q}}\left|x_{n}-x_{m}\right|$ where $\tilde{q}=\prod_{i=1}^{l} q_{i}$. The total number of branch ends is also $\tilde{q}$. To terminate all the branches $\tilde{q}$ factors of the form $\zeta_{m_{i}}^{000}$ (bounded by (5.20)) should be multiplied resulting in the a term that multiplies the bound on a sum of the form

$$
\begin{aligned}
& \sum_{\left\{m_{i}\right\}} \exp \left[-\frac{\left(\gamma-\varepsilon^{\prime}\right)}{\tilde{q}} s \sum_{i}\left|x_{n}-x_{m_{i}}\right|-\left(\gamma-\varepsilon^{\prime}\right) s \sum_{i}\left|x_{m_{i}}\right|\right] \\
& \leq \exp -\frac{\left(\gamma-\varepsilon^{\prime}\right)}{\tilde{q}} s \sum_{i}\left|x_{n}\right| \sum_{\left\{m_{i}\right\}} \exp -\left(1-\frac{1}{\tilde{q}}\right)\left(\gamma-\varepsilon^{\prime}\right) s \sum_{i}\left|x_{m_{i}}\right| \\
& =e^{-\frac{\gamma-\varepsilon^{\prime}}{\tilde{q}} s \tilde{q}\left|x_{n}\right|} \sum_{\left\{m_{i}\right\}} \exp -\left(1-\frac{1}{\tilde{q}}\right)\left(\gamma-\varepsilon^{\prime}\right) s \sum_{i}\left|x_{m_{i}}\right| \equiv S_{\varepsilon} e^{-\left(\gamma-\varepsilon^{\prime}\right) s\left|x_{n}\right|}
\end{aligned}
$$

restoring the original convergence rate. As the product consists of $k$ terms the evaluation of $\left|\zeta_{n}^{m_{1} m_{2} m_{3}}\right|^{s k}$ is required for the use of the Hölder inequality. Therefore it is required that $0<s<1 / 2 k$.

Lemma 9. For a given $k$ (the number of $\zeta^{\prime} s$ ), $\delta, \varepsilon, \varepsilon^{\prime}, \eta^{\prime}>0$ and $0<s<\frac{1}{2 k}$

$$
\left\langle\left|\sum_{\left\{m_{i}\right\}} \zeta_{n}^{m_{1} m_{2} m_{3}} \zeta_{m_{1}}^{m_{4} m_{5} m_{6}} \cdots \zeta_{m_{N-1}}^{000}\right|^{s}\right\rangle_{\delta} \leq F_{\delta}^{(k)} e^{-\left(\gamma-\varepsilon-\varepsilon^{\prime}\right) s\left|x_{n}\right|}
$$

or using the Chebyshev inequality (5.15)

$$
\operatorname{Pr}\left(\left|\sum_{\left\{m_{i}\right\}} \zeta_{n}^{m_{1} m_{2} m_{3}} \zeta_{m_{1}}^{m_{4} m_{5} m_{6}} \cdots \zeta_{m_{N-1}}^{000}\right| \geq\left(F_{\delta}^{(k)}\right)^{1 / s} e^{-\left(\gamma-\varepsilon-\varepsilon^{\prime}-\eta^{\prime}\right)\left|x_{n}\right|}\right) \leq e^{-\eta^{\prime} s\left|x_{n}\right|},
$$

where $F_{\delta}^{(k)}$ is a constant which is built iteratively by the construction demonstrated in (5.23) and (5.25) and is proportional to $D_{\delta}$.

It is of importance, that any product with the same number of zetas has the same bound with the same probability. This allows us to bound $c_{n}^{(k)}$ by counting the number of different configurations, $R_{k}$, of the product for a given $k$ and then multiplying it by the bound of each product. This proves the theorem:

Theorem 10. For a given $k$ and $\delta, \varepsilon, \varepsilon^{\prime}, \eta^{\prime}>0$

$$
\operatorname{Pr}\left(\left|c_{n}^{(k)}\right| \geq\left(F_{\delta}^{(k)}\right)^{k} e^{c k^{2}+c^{\prime} k} e^{-\left(\gamma-\varepsilon-\varepsilon^{\prime}-\eta^{\prime}\right)\left|x_{n}\right|}\right) \leq e^{-c^{\prime}} e^{-\eta^{\prime}\left|x_{n}\right| / k} .
$$


where $F_{\delta}^{(k)}$ which is proportional to $D_{\delta}$ and $c$ and $c^{\prime}$ are constants.

Proof. Using Lemma 9 and summing over configurations denoted by $i_{c}$ one obtains the bound

$$
\begin{aligned}
\left\langle\left|c_{n}^{(k)}\right|^{s}\right\rangle_{\delta} & =\left\langle\left|\sum_{i_{c}=1}^{R_{k}}\left\{\sum_{\left\{m_{i}\right\}} \zeta_{n}^{m_{1} m_{2} m_{3}} \zeta_{m_{1}}^{m_{4} m_{5} m_{6}} \cdots \zeta_{m_{k-1}}^{000}\right\}\right|^{s}\right\rangle_{\delta} \\
& \leq \sum_{i_{c}=1}^{R_{k}}\left\langle\left|\sum_{\left\{m_{i}\right\}} \zeta_{n}^{m_{1} m_{2} m_{3}} \zeta_{m_{1}}^{m_{4} m_{5} m_{6}} \cdots \zeta_{m_{k-1}}^{000}\right|^{s}\right\rangle_{\delta} \leq R_{k} F_{\delta}^{(k)} e^{-\left(\gamma-\varepsilon-\varepsilon^{\prime}\right) s\left|x_{n}\right|}
\end{aligned}
$$

Using (4.6)

$$
\left\langle\left|c_{n}^{(k)}\right|^{s}\right\rangle_{\delta} \leq e^{2 k} F_{\delta}^{(k)} e^{-\left(\gamma-\varepsilon-\varepsilon^{\prime}\right) s\left|x_{n}\right|}
$$

or

$$
\operatorname{Pr}\left(\left|c_{n}^{(k)}\right| \geq e^{c^{\prime} / s}\left(F_{\delta}^{(k)}\right)^{1 / s} e^{-\left(\gamma-\varepsilon-\varepsilon^{\prime}-\eta^{\prime}\right)\left|x_{n}\right|} e^{2 k / s}\right) \leq e^{-c^{\prime}} e^{-\eta^{\prime} s\left|x_{n}\right|}
$$

Choosing the largest $s$ produces a bound where $c, c^{\prime}$ are constants and $F_{\delta}^{(k)}$ is a constant which is built iteratively by the construction demonstrated in (5.23) and (5.25) and is proportional to $D_{\delta}$.

Remark 11. From (4.6) one sees that $c \simeq 2$ and later we set $c^{\prime}=c N$.

\section{Numerical Results}

In this section we will check how well the perturbation series up to the second order in $\beta$ approximates the numerical solution of (1.1). For this purpose we use the expressions for $c_{n}^{(1)}$ and $c_{n}^{(2)}$ which were obtained in (3.12) and (3.19), respectively, and also the expression for the renormalized energies $E_{n}^{\prime}$ up to second order in $\beta$ which are given by (3.18). We use the perturbation expansion up to the second order in $\beta$, namely

$$
\bar{c}_{n}=c_{n}^{(0)}+\beta c_{n}^{(1)}+\left.\beta^{2} c_{n}^{(2)}\right|_{\beta=0},
$$

where we took $\left.c_{n}^{(2)}\right|_{\beta=0}$ in order to keep only the contribution of $c_{n}^{(2)}$ up to the order $\beta^{2}$. To compare, we plot the real and the imaginary parts of both the numerical solution of (1.1) with the distribution (5.12) and the perturbative approximation $\bar{c}_{n}$. From figures 6.1 and 6.2 we see that the correspondence between the numerical solution of (1.1) and the perturbative approximation is good for times $<50$ for $\beta=0.1$ and times $<200$ for $\beta=0.01$. Additionally, the correspondence of the central site, $c_{0}$, which is used as the initial condition, $c_{n}(t=0)=\delta_{n 0}$, is much better than the correspondence of the neighboring sites. A possible explanation for this could be that the nonlinear perturbation is more pronounced at the states $n$ with $c_{n}(t=0)=0$, this is due to the fact that for $\beta=0$ those sites are unpopulated (zero) for all times, resulting in lower signal to noise ratio. To examine the convergence in time we will define a time, $t^{*}$. For times $t<t^{*}$ the relative difference between the $L_{2}$ norms of the exact and the approximate solutions, $d_{2}(t)$, defined bellow, is less than $10 \%$. It is instructive to introduce the following definitions. 

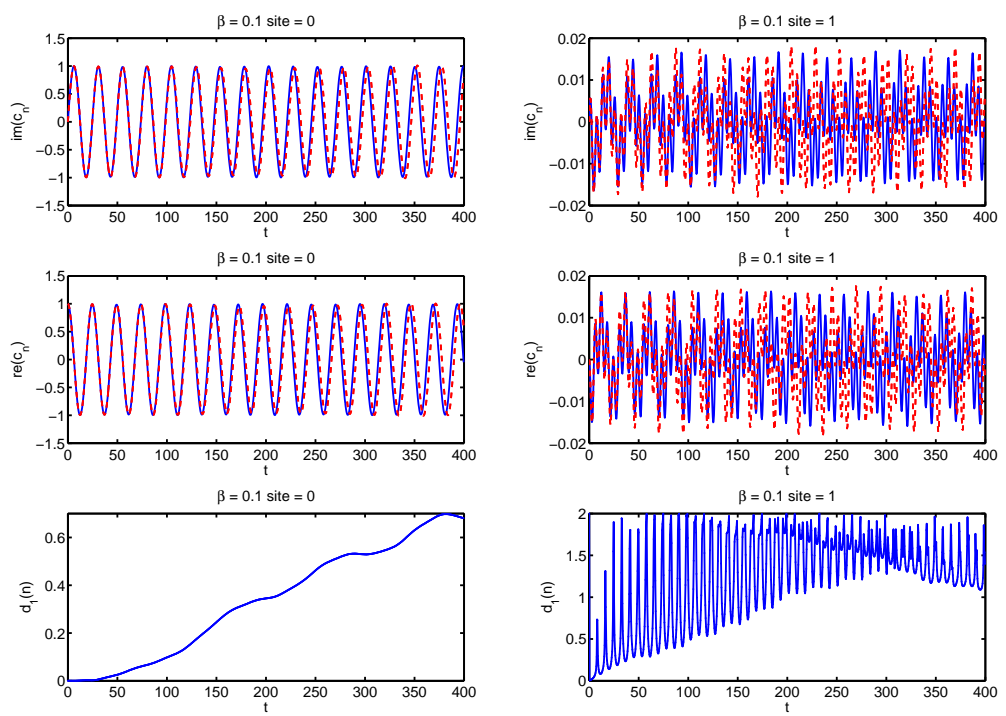

FiguRE 6.1. In the first and the second rows of the figure are presented the imaginary and real parts, respectively, of the numerical solution of (1.1) (blue, solid) and the perturbative approximation (red, dashed) as function of time for $c_{0}$ (left column) and $c_{1}$ (right column). In the third row the relative difference, $d_{1}(t)$, as defined in Definition 12 is plotted. The parameters of the plot are, $J=0.25, \Delta=1$ and $\beta=0.1$ and lattice size of 128. See (1.1) for the definition of the constants.

Definition 12. The relative difference between the exact and the perturbative solution at site $n$ is defined as

$$
d_{1}(t)=\frac{2\left|c_{n}(t)-\bar{c}_{n}(t)\right|}{\left|c_{n}(t)\right|+\left|\bar{c}_{n}(t)\right|}
$$

Definition 13. $t^{*}$ is a time until which the relative difference between the exact and the perturbative solution, $d_{2}(t)$, is less than $10 \%$.

$$
d_{2}(t)=\frac{\sum_{n}\left|c_{n}(t)-\bar{c}_{n}(t)\right|^{2}}{\sum_{n}\left|c_{n}(t)\right|^{2}} \leq 0.1 .
$$

In figure 6.3 we see that as $\beta$ becomes smaller the time for which the expansion to second order in $\beta$ is close to the exact solution (within $10 \%$ ), $t^{*}$, increases. 

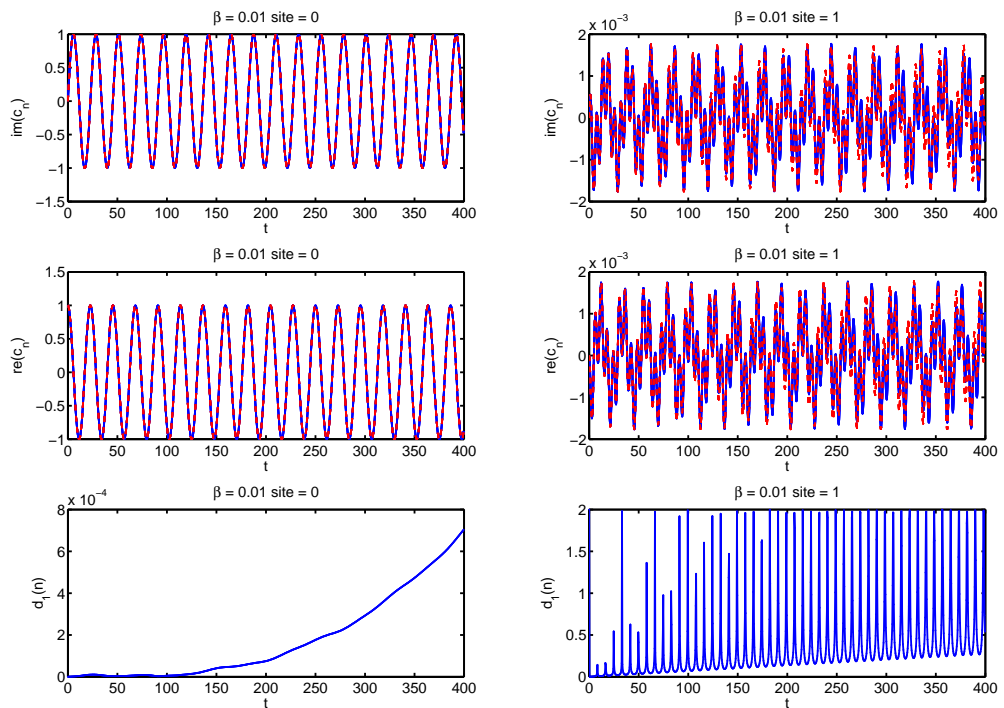

Figure 6.2. Same as Figure 6.1 but for $\beta=0.01$.

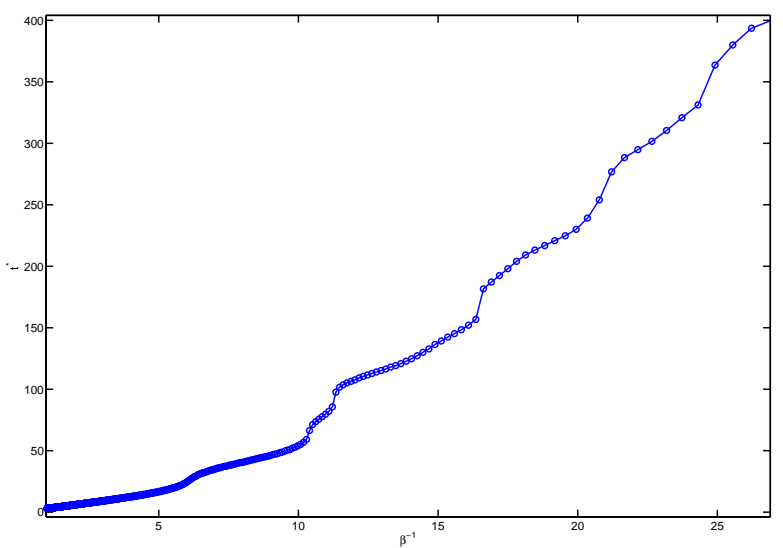

FigURE 6.3. $t^{*}$ (see text) as a function of the inverse nonlinearity strength, $\beta^{-1}$.

\section{BOUNDING THE REMAINDER}

In order to bound the solution we have to bound, $Q_{n}$, the remainder of the expansion (2.13)

$$
c_{n}(t)=c_{n}^{(0)}+\beta c_{n}^{(1)}+\beta^{2} c_{n}^{(2)}+\cdots+\beta^{N-1} c_{n}^{(N-1)}+\beta^{N} Q_{n} .
$$

This is achieved applying the bootstrap argument to the remainder. Substituting the expansion (2.13) into the equation (3.4) and writing a differential equation for 
the remainder gives

$$
\begin{aligned}
i \partial_{t} Q_{n} & =\sum_{m_{1} m_{2} m_{3}} \sum_{N-1 \leq k+l+q \leq 3(N-1)}^{N-1} \beta^{k+l+q+1-N} V_{n}^{m_{1} m_{2} m_{3}} c_{m_{1}}^{*(q)} c_{m_{2}}^{(l)} c_{m_{3}}^{(k)} e^{i\left(E_{n}^{\prime}+E_{m_{1}}^{\prime}-E_{m_{2}}^{\prime}-E_{m_{3}}^{\prime}\right) t} \\
& +\sum_{m_{1} m_{2} m_{3}} \sum_{0 \leq k+l \leq 2(N-1)}^{N-1} \beta^{l+k+1} V_{n}^{m_{1} m_{2} m_{3}} Q_{m_{1}}^{*} c_{m_{2}}^{(l)} c_{m_{3}}^{(k)} e^{i\left(E_{n}^{\prime}+E_{m_{1}}^{\prime}-E_{m_{2}}^{\prime}-E_{m_{3}}^{\prime}\right) t} \\
& +2 \sum_{m_{1} m_{2} m_{3}} \sum_{0 \leq k+l \leq 2(N-1)}^{N-1} \beta^{l+k+1} V_{n}^{m_{1} m_{2} m_{3}} Q_{m_{3}} c_{m_{1}}^{(l) *} c_{m_{2}}^{(k)} e^{i\left(E_{n}^{\prime}+E_{m_{1}}^{\prime}-E_{m_{2}}^{\prime}-E_{m_{3}}^{\prime}\right) t} \\
& +2 \sum_{m_{1} m_{2} m_{3}} \sum_{k=0}^{N-1} \beta^{N+k+1} V_{n}^{m_{1} m_{2} m_{3}} Q_{m_{1}}^{*} Q_{m_{2}} c_{m_{3}}^{(k)} e^{i\left(E_{n}^{\prime}+E_{m_{1}}^{\prime}-E_{m_{2}}^{\prime}-E_{m_{3}}^{\prime}\right) t} \\
& +\sum_{m_{1} m_{2} m_{3}} \sum_{k=0}^{N-1} \beta^{N+k+1} V_{n}^{m_{1} m_{2} m_{3}} c_{m_{1}}^{(k) *} Q_{m_{2}} Q_{m_{3}} e^{i\left(E_{n}^{\prime}+E_{m_{1}}^{\prime}-E_{m_{2}}^{\prime}-E_{m_{3}}^{\prime}\right) t} \\
& +\sum_{m_{1} m_{2} m_{3}} \beta^{2 N+1} V_{n}^{m_{1} m_{2} m_{3}} Q_{m_{1}}^{*} Q_{m_{2}} Q_{m_{3}} e^{i\left(E_{n}^{\prime}+E_{m_{1}}^{\prime}-E_{m_{2}}^{\prime}-E_{m_{3}}^{\prime}\right) t}
\end{aligned}
$$

where the sums over orders are understood as follows. By $\sum_{N-1 \leq k+l+q \leq 3(N-1)}^{N-1}$ we mean $\sum_{k, l, q=0}^{N-1}$ with the constraint $N-1 \leq k+l+q$. Integrating and using the fact, $Q_{n}(t=0)=0$,

$$
\begin{aligned}
\frac{\left|Q_{n}\right|}{t} & \leq \sum_{m_{1} m_{2} m_{3}} \sum_{N-1 \leq k+l+q \leq 3(N-1)}^{N-1} \beta^{k+l+q+1-N}\left|V_{n}^{m_{1} m_{2} m_{3}}\right| \sup _{0 \leq t^{\prime} \leq t}\left[\left|c_{m_{1}}^{*(k)}\right|\left|c_{m_{2}}^{(l)}\right|\left|c_{m_{3}}^{(q)}\right|\right] \\
& +\sum_{m_{1} m_{2} m_{3}} \sum_{0 \leq k+l \leq 2(N-1)}^{N-1} \beta^{l+k+1}\left|V_{n}^{m_{1} m_{2} m_{3}}\right| \sup _{0 \leq t^{\prime} \leq t}\left[\left|Q_{m_{1}}^{*}\right|\left|c_{m_{2}}^{(l)}\right|\left|c_{m_{3}}^{(k)}\right|\right] \\
& +2 \sum_{m_{1} m_{2} m_{3}} \sum_{0 \leq k+l \leq 2(N-1)}^{N-1} \beta^{l+k+1}\left|V_{n}^{m_{1} m_{2} m_{3}}\right| \sup _{0 \leq t^{\prime} \leq t}\left[\left|Q_{m_{3}}\right|\left|c_{m_{1}}^{(l) *}\right|\left|c_{m_{2}}^{(k)}\right|\right] \\
& +2 \sum_{m_{1} m_{2} m_{3}} \sum_{k=0}^{N-1} \beta^{N+k+1}\left|V_{n}^{m_{1} m_{2} m_{3}}\right| \sup _{0 \leq t^{\prime} \leq t}\left[\left|Q_{m_{1}}^{*}\right|\left|Q_{m_{2}}\right|\left|c_{m_{3}}^{(k)}\right|\right] \\
& +\sum_{m_{1} m_{2} m_{3}} \sum_{k=0}^{N-1} \beta^{N+k+1} \mid V_{n}^{m_{1} m_{2} m_{3} \mid} \sup _{0 \leq t^{\prime} \leq t}\left[\left|Q_{m_{2}}\right|\left|Q_{m_{3}}\right|\left|c_{m_{1}}^{(k) *}\right|\right] \\
& +\beta^{2 N+1} \sum_{m_{1} m_{2} m_{3}} \mid V_{n}^{m_{1} m_{2} m_{3} \mid} \sup _{0 \leq t^{\prime} \leq t}\left[\left|Q_{m_{1}}^{*}\right|\left|Q_{m_{2}}\right|\left|Q_{m_{3}}\right|\right]
\end{aligned}
$$

where $t$ is the upper limit of the integration.

Since $Q_{n}(t)$ is continuous and $Q_{n}(t=0)=0$, for small $t, Q_{n}(t) \sim t \cdot S_{1}$. Therefore we can always find a sufficiently small $\tau>0$, such that

$$
\left|Q_{n}(\tau)\right|<2 \tau \cdot S_{1}
$$


where

$(7.5)$

$S_{1}=\sum_{m_{1} m_{2} m_{3}} \sum_{N-1 \leq k+l+q \leq 3(N-1)}^{N-1} \beta^{k+l+q+1-N}\left|V_{n}^{m_{1} m_{2} m_{3}}\right| \sup _{0 \leq t^{\prime} \leq t}\left[\left|c_{m_{1}}^{*(k)}\right|\left|c_{m_{2}}^{(l)}\right|\left|c_{m_{3}}^{(q)}\right|\right]$.

Assume, that there is some time, $t$, for which,

$$
\left|Q_{n}(t)\right|>2 t \cdot S_{1},
$$

than since $Q_{n}(t)$ is continuous and (7.4) holds there is a time, $t_{0}$, where

$$
\left|Q_{n}\left(t_{0}\right)\right|=2 t_{0} \cdot S_{1} .
$$

Inserting this equality into the inequality (7.3), we get an interval $0 \leq t \leq t_{0}$ for which (7.4) holds (see (7.25)). We proceed by bounding $S_{1}$ and other sums of (7.3).

Using the Theorem 10 obtained in the end of the Section 5, we can bound, $S_{1}$. The inequality is violated with a probability found from (5.29). We start with the bound

$$
\begin{aligned}
& S_{1} \leq \sum_{\left\{m_{i}\right\}} \sum_{N-1 \leq k+l+q \leq 3(N-1)}^{N-1} \beta^{k+l+q+1-N}\left|V_{n}^{m_{1} m_{2} m_{3}}\right| \times \\
& \times e^{c^{\prime}(k+l+q)+c\left(k^{2}+l^{2}+q^{2}\right)} e^{-\left(\gamma-\varepsilon^{\prime}-\eta^{\prime}\right)\left(\left|x_{m_{1}}\right|+\left|x_{m_{2}}\right|+\left|x_{m_{3}}\right|\right)}
\end{aligned}
$$

we find from (2.11)

$$
\begin{aligned}
& \sum_{m_{1} m_{2} m_{3}}\left|V_{n}^{m_{1} m_{2} m_{3}}\right| e^{-\left(\gamma-\varepsilon^{\prime}-\eta^{\prime}\right)\left(\left|x_{m_{1}}\right|+\left|x_{m_{2}}\right|+\left|x_{m_{3}}\right|\right)} \\
& \leq V_{\delta}^{\varepsilon, \varepsilon^{\prime}} \sum_{m_{1} m_{2} m_{3}} e^{\varepsilon\left(\left|x_{n}\right|+\left|x_{m_{1}}\right|+\left|x_{m_{2}}\right|+\left|x_{m_{3}}\right|\right)} \\
& \times e^{-\frac{1}{3}\left(\gamma-\varepsilon^{\prime}\right)\left(\left|x_{n}-x_{m_{1}}\right|+\left|x_{n}-x_{m_{2}}\right|+\left|x_{n}-x_{m_{3}}\right|\right)} e^{-\left(\gamma-\eta^{\prime}-\varepsilon^{\prime}\right)\left(\left|x_{m_{1}}\right|+\left|x_{m_{2}}\right|+\left|x_{m_{3}}\right|\right)} \\
& \leq V_{\delta}^{\varepsilon, \varepsilon^{\prime}} e^{-\left(\gamma-\varepsilon-\varepsilon^{\prime}\right)\left|x_{n}\right|} \sum_{m_{1} m_{2} m_{3}} e^{-\frac{2}{3}\left(\gamma-\eta^{\prime}-\varepsilon\right)\left(\left|x_{m_{1}}\right|+\left|x_{m_{2}}\right|+\left|x_{m_{3}}\right|\right)}=C_{\delta}^{\gamma, \varepsilon, \varepsilon^{\prime}, \eta^{\prime}} e^{-\left(\gamma-\varepsilon-\varepsilon^{\prime}\right)\left|x_{n}\right|}
\end{aligned}
$$

for $\frac{\gamma}{3}<\left(\gamma-\eta^{\prime}\right)$. Therefore for $\eta^{\prime}$ sufficiently small, substituting back we get

$$
\begin{aligned}
S_{1} & \leq C_{\delta}^{\gamma, \varepsilon, \varepsilon^{\prime \prime}, \eta^{\prime}} e^{-\left(\gamma-\varepsilon-\varepsilon^{\prime}\right)\left|x_{n}\right|} \sum_{N-1 \leq k+l+q \leq 3(N-1)}^{N-1} \beta^{k+l+q+1-N} e^{c\left(k^{2}+l^{2}+q^{2}\right)} e^{c^{\prime}(k+l+q)} \\
& \equiv C_{\delta}(N) e^{6 c N^{2}} e^{-\left(\gamma-\varepsilon-\varepsilon^{\prime}\right)\left|x_{n}\right|},
\end{aligned}
$$

where we used $c^{\prime}=c N$, and $C_{\delta}(N)=O\left(N^{3}\right)$. For the bound (7.10) to be violated at least one of the $c_{m}^{(k)}$ has to satisfy the inequality (5.29) and the probability for this is bounded by $e^{-c^{\prime}} e^{-\eta^{\prime}\left|x_{m}\right| / k}$. Therefore the probability that (7.10) will be violated is bounded by

$$
e^{-c^{\prime}} \sum_{k=1}^{N-1} \sum_{n=-\infty}^{\infty} e^{-\eta^{\prime}\left|x_{n}\right| / k}=e^{-c^{\prime}} \sum_{k=1}^{N-1}\left(\frac{2}{1-e^{-\eta^{\prime} / k}}-1\right) \sim \sum_{k=1}^{N-1} \frac{k}{\eta^{\prime}} \sim \frac{N^{2}}{\eta^{\prime}} e^{-c N},
$$


where we have expanded the exponent $e^{-\eta^{\prime} / k}$ using the fact that for large $N$ the sum is dominated by terms with $k \gg 1$ and $\eta^{\prime} / k \ll 1$. Setting $c^{\prime}=N$ provides the convergence of the probability with the expansion order, i.e.,

$$
\operatorname{Pr}\left(\begin{array}{c}
\sum_{m_{1} m_{2} m_{3}} \sum_{N-1 \leq k+l+q \leq 3(N-1)}^{N-1} \beta^{k+l+q+1-N}\left|V_{n}^{m_{1} m_{2} m_{3}}\right|\left|c_{m_{1}}^{*(k)}\right|\left|c_{m_{2}}^{(l)}\right|\left|c_{m_{3}}^{(q)}\right|
\end{array}\right) \leq \frac{\text { const }}{\eta^{\prime}} N^{2} e^{-c N} .
$$

Now we turn to find the point $t_{0}$ defined in (7.7). To bound other expressions in (7.3), we use

$$
\left|Q_{m}\left(t_{0}\right)\right|=2 t_{0} \cdot S_{1} \leq M\left(t_{0}\right) e^{-\nu\left|x_{m}\right|},
$$

where following (7.12),

$$
M\left(t_{0}\right):=2 t_{0} C_{\delta}(N) e^{6 c N^{2}}
$$

with $\nu=\gamma-\varepsilon-\varepsilon^{\prime}$. In what follows, unless stated differently, $M$ will mean $M\left(t_{0}\right)$.

First we will bound the linear term in the $Q_{n}$ in (7.3). The sum over $m_{2}$ and $m_{3}$ is bounded similarly to the sums in the inhomogeneous term and the sum over $m_{1}$ is bounded using the bootstrap assumption (7.13), resulting in

$$
\begin{aligned}
S_{2}= & \sum_{m_{2} m_{3}} \sum_{0 \leq k+l \leq 2(N-1)}^{N-1} \beta^{l+k+1} \sum_{m_{1}}\left|V_{n}^{m_{1} m_{2} m_{3}}\right|\left|Q_{m_{1}}^{*}\right|\left|c_{m_{2}}^{(l)}\right|\left|c_{m_{3}}^{(k)}\right| \\
& \leq M \sum_{m_{2} m_{3}} \sum_{0 \leq k+l \leq 2(N-1)}^{N-1} \beta^{l+k+1} e^{c\left(k^{2}+l^{2}\right)} e^{c^{\prime}(k+l)} \sum_{m_{1}}\left|V_{n}^{m_{1} m_{2} m_{3}}\right| e^{-\nu\left|x_{m_{1}}\right|} e^{-\left(\gamma-\eta^{\prime}-\varepsilon^{\prime}\right)\left(\left|x_{m_{2}}\right|+\left|x_{m_{3}}\right|\right)}
\end{aligned}
$$

for $\frac{1}{3}\left(\gamma-\varepsilon^{\prime}\right)<\nu$ and $\frac{\gamma}{3}<\gamma-\eta^{\prime}$. This is similar to the sum in equation (7.9) and gives a result with a similar dependence on $\left|x_{n}\right|$,

$$
\begin{aligned}
S_{2} & \leq \sum_{m_{2} m_{3}} \sum_{0 \leq k+l \leq 2(N-1)}^{N-1} \beta^{l+k+1} \sum_{m_{1}}\left|V_{n}^{m_{1} m_{2} m_{3}}\right|\left|Q_{m_{1}}^{*}\right|\left|c_{m_{2}}^{(l)}\right|\left|c_{m_{3}}^{(k)}\right| \\
& \leq C_{\delta}(N) e^{2 c N^{2}} M e^{-\left(\gamma-\varepsilon-\varepsilon^{\prime}\right)\left|x_{n}\right|} .
\end{aligned}
$$

with the same probability as in (7.11). Therefore the linear term in $Q_{n}$ is bounded by the probabilistic bound

$$
\operatorname{Pr}\left(\begin{array}{c}
\sum_{m_{1} m_{2} m_{3} ; l, k} \beta^{l+k+1}\left|V_{n}^{m_{1} m_{2} m_{3}}\right|\left|Q_{m_{1}}^{*}\right|\left|c_{m_{2}}^{(l)}\right|\left|c_{m_{3}}^{(k)}\right| \\
\geq \beta C_{\delta}(N) e^{4 c N^{2}} M e^{-\left(\gamma-\varepsilon-\varepsilon^{\prime}-\eta^{\prime}\right)\left|x_{n}\right|}
\end{array}\right) \leq \frac{\text { const }}{\eta^{\prime}} N^{2} e^{-c N}
$$

with $C_{\delta}=O\left(N^{2}\right)$. A similar bound is found for the third term on RHS of (7.3).

The fourth sum of equation (7.3)

$$
S_{4}=\sum_{m_{1} m_{2} m_{3} ; k} \beta^{N+k+1}\left|V_{n}^{m_{1} m_{2} m_{3}}\right|\left|Q_{m_{1}}^{*}\right|\left|Q_{m_{2}}\right|\left|c_{m_{3}}^{(k)}\right|
$$

is bounded by

$$
\operatorname{Pr}\left(\begin{array}{c}
\sum_{m_{1} m_{2} m_{3} ; k} \beta^{N+k+1}\left|V_{n}^{m_{1} m_{2} m_{3}}\right|\left|Q_{m_{1}}^{*}\right|\left|Q_{m_{2}}\right|\left|c_{m_{3}}^{(k)}\right| \\
\geq C_{\delta}(N) e^{2 c N^{2}} \beta^{N+1} M^{2} e^{-\left(\gamma-\varepsilon-\varepsilon^{\prime}-\eta^{\prime}\right)\left|x_{n}\right|}
\end{array}\right) \leq \frac{\text { const }}{\eta^{\prime}} N^{2} e^{-c N}
$$


with $C_{\delta}=O(N)$. The last term in (7.3) is

$$
S_{5}=\beta^{2 N+1} \sum_{m_{1} m_{2} m_{3}}\left|V_{n}^{m_{1} m_{2} m_{3}}\right|\left|Q_{m_{1}}^{*}\right|\left|Q_{m_{2}}\right|\left|Q_{m_{3}}\right| .
$$

and it is bounded by

$$
S_{5} \leq \beta^{2 N+1} M^{3} e^{-\left(\gamma-\varepsilon-\varepsilon^{\prime}\right)\left|x_{n}\right|} .
$$

To summarize, substitution of the equality (7.13) in (7.3) to find a time $t_{0}$ for which assumption (7.6) is valid results in the following inequality which is satisfied with the probability that is the sum of the probabilities given by the RHS of (7.12), (7.17), (7.19),

$$
2 t_{0} S_{1} \leq t_{0} \cdot S_{1}+t_{0} C_{\delta}(N)\left(e^{6 c N^{2}}+2 \beta e^{4 c N^{2}} M+4 \beta^{N+1} e^{2 c N^{2}} M^{2}+8 \beta^{2 N+1} M^{3}\right) e^{-\left(\gamma-\varepsilon-\varepsilon^{\prime}\right)\left|x_{n}\right|},
$$

with $C_{\delta}=O\left(N^{3}\right)$. Multiplying by $e^{\left(\gamma-\varepsilon-\varepsilon^{\prime}\right)\left|x_{n}\right|}$ both sides of the inequality (7.22) and taking the infimum with respect to $n$, gives

$$
\inf _{n}\left(S_{1} e^{\left(\gamma-\varepsilon-\varepsilon^{\prime}\right)\left|x_{n}\right|}\right) \leq 2 C_{\delta}(N)\left(\beta e^{4 c N^{2}} M+2 \beta^{N+1} e^{2 c N^{2}} M^{2}+4 \beta^{2 N+1} M^{3}\right) .
$$

Setting $A_{N}:=e^{2 c N^{2}}, \tilde{C}:=\inf _{n}\left(S_{1} e^{\left(\gamma-\varepsilon-\varepsilon^{\prime}\right)\left|x_{n}\right|}\right)$ and using the definition of $M$ (see $(7.14))$

$$
\tilde{C} \leq 2 \beta t_{0} \cdot A_{N}^{5} C_{\delta}+4 \beta^{N+1} t_{0}^{2} A_{N}^{7} C_{\delta}^{2}+8 \beta^{2 N+1} t_{0}^{3} A_{N}^{9} .
$$

For sufficiently small $\beta t_{0}$ the first term on the RHS of (7.24) is dominant and therefore,

$$
t_{0} \geq \frac{\tilde{C}}{2 \beta A_{N}^{5} C_{\delta}}
$$

Note, that $\tilde{C}>0$, since it is an infimum of a sum of positive quantities, however we do not calculate it explicitly in this paper, nevertheless it is likely to be of the order of $C_{\delta} A_{N}^{3}$. This proves that,

$$
\left|Q_{n}(t)\right| \leq M(t) \cdot e^{-\left(\gamma-\varepsilon-\varepsilon^{\prime}\right)\left|x_{n}\right|}=2 t \cdot C_{\delta} e^{6 c N^{2}} e^{-\left(\gamma-\varepsilon-\varepsilon^{\prime}\right)\left|x_{n}\right|}
$$

for times $t \leq t_{0}$, where $M(t)$ is given by extending the definition (7.14) by replacing $t_{0}$ by $t$.

Theorem 14. For $t=O\left(\beta^{-1}\right)$ with $c \cong 2$, and assuming that Conjecture 3 holds

$$
\operatorname{Pr}\left(\left|Q_{n}\right| \geq 2 t \cdot C_{\delta} e^{6 c N^{2}} e^{-\left(\gamma-\varepsilon-\varepsilon^{\prime}\right)\left|x_{n}\right|}\right) \leq \frac{\text { const }}{\eta^{\prime}} N^{2} e^{-c N}
$$

The bound on the probability of statement (7.26) is calculated by summing the RHS of $((7.12),(7.17),(7.19))$. The contribution of the remainder term is

$$
\left|\beta^{N} Q_{n}\right| \leq \text { const } \cdot e^{6 c N^{2}+N \ln \beta+\ln t} e^{-\left(\gamma-\varepsilon-\varepsilon^{\prime}\right)\left|x_{n}\right|} .
$$

Note that for a given $t$ and $\beta$ there is an optimum $N$ for which the remainder is minimal. Additionally, for any fixed time and order $N, \lim _{\beta \rightarrow 0}\left|\beta^{N} Q_{n}\right| / \beta^{N-1}=0$, which shows that the series is in fact an asymptotic one [63]. 


\section{Summary}

In this paper a perturbation expansion in powers of $\beta$ was developed (Sections $2,3)$ for the solution of the NLSE with a random potential. It required the removal of the secular terms for this problem. To best of our knowledge it is the first time it was done for a multivariate problem. The quality of the expansion to the second order was tested in Section 6. In Section 4 it was shown that the number of terms grows exponentially with the order. In Section 5 a probabilistic bound on the general term (5.29) was derived. It relies on the Conjecture 3. The resulting bound was tested numerically. Finally, a bound on the remainder was obtained for a finite time, showing that the series is asymptotic. For time shorter than $t_{0}$ which is given by (7.25) there is a front $\bar{x}(t) \propto \ln t$ such that for $x_{n}>\bar{x}(t)$ both the remainder, $\beta^{N} Q_{n}(t)$ and $c_{n}(t)$ are exponentially small.

The work leaves several open problems that should be subject of further research:

(1) Turning the perturbation theory developed in the present work into a practical method for solution of the NLSE and similar nonlinear differential equations. The control on the error should be obtained using the methods presented in Sec. 7.

(2) Can the front $\bar{x}(t) \propto \ln t$ be found for arbitrarily long times?

(3) The asymptotic nature of the series. Is it just an asymptotic series or a convergent one?

(4) If the series is asymptotic can it be resummed ?

(5) The $e^{c k^{2}+c^{\prime} k}$ in (5.29) results from the repeated use of the Hölder inequality and a very generous bound on the probability distributions. An effort should be made to improve it.

(6) There are various properties of the Anderson model that have been used here. Some of them were tested numerically. It would be of great value if Conjecture 3, Corollary 4 and Conjecture 5 were rigorously established, even at the limit of a strong disorder. In the present work we rely only on Corollary 4 (that was tested numerically, see Fig. 5.1). The rigorous proof of the unimodality of $\gamma(E)$ for the uniform distribution of the random potentials, $\varepsilon_{x}$, may also be useful.

(7) It would be very useful if Conjecture 6 could be rigorously obtained.

We enjoyed many extensive illuminating and extremely critical discussions with Michael Aizenman. We also had informative discussions with S. Aubry, V. Chulaevski, S. Flach, I. Goldshield, M. Goldstein, I. Guarneri, M. Sieber, W.-M. Wang and S. Warzel. This work was partly supported by the Israel Science Foundation (ISF), by the US-Israel Binational Science Foundation (BSF), by the USA National Science Foundation (NSF), by the Minerva Center of Nonlinear Physics of Complex Systems, by the Shlomo Kaplansky academic chair, by the Fund for promotion of research at the Technion and by the E. and J. Bishop research fund. The work was done partially while SF was visiting the Institute of Mathematical Sciences, National University of Singapore in 2006 and the Center of Nonlinear Physics of Complex Systems in Dresden in 2007 and while YK visited the department of Mathematics at Rutgers University and while AS was visiting the Lewiner Institute of Theoretical Physics at the Technion in 2007. The visits were supported in part by these Institutes. 


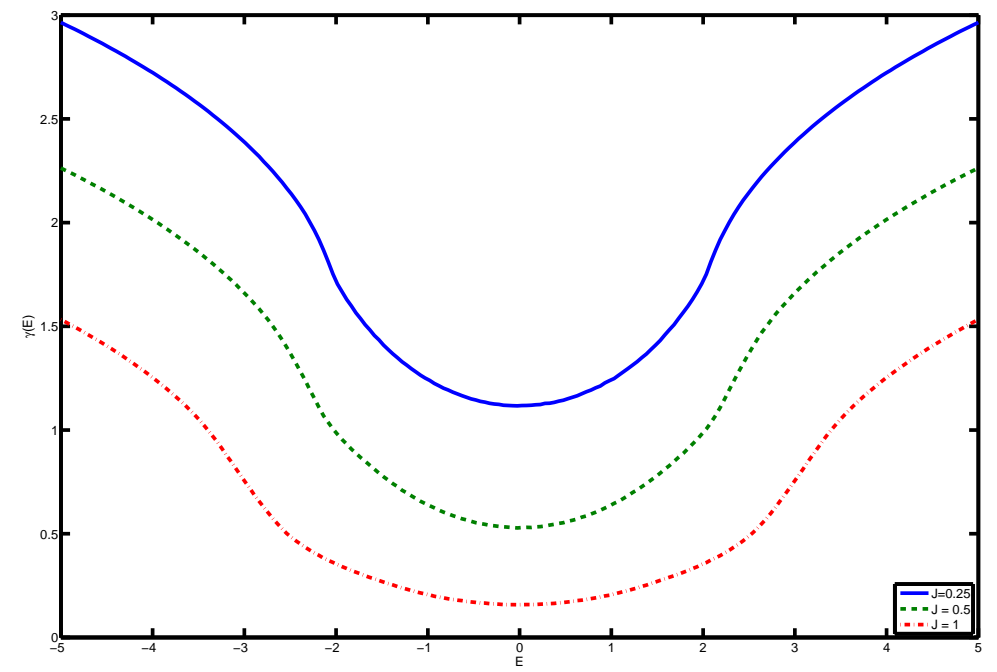

Figure 8.1. The Lyapunov exponent as a function of energy for the uniform distribution defined in (5.12). The solid (blue) line is for $J=0.25$, the dashed (green) line is for $J=0.5$ and the dot-dashed line (red) is for $J=1$.

\section{APPENDIX}

The average Lyapunov exponent was calculated numerically using the transfer matrix technique for a uniform distribution defined in (5.12). The results are presented in Fig. 8.1. It can be seen that $\gamma(E)$ is unimodal.

\section{REFERENCES}

[1] C. Sulem and P. L. Sulem. The nonlinear Schrödinger equation self-focusing and wave collapse. Springer, 1999.

[2] D. J. Thouless. Relation between density of states and range of localization for one dimensional random systems. J. Phys. C: Solid State Phys., 5(1):77, 1972.

[3] G. P. Agrawal. Nonlinear fiber optics, volume 4th. Academic Press, Burlington, MA ; London, 2007.

[4] F. Dalfovo, S. Giorgini, L. P. Pitaevskii, and S. Stringari. Theory of Bose-Einstein condensation in trapped gases. Rev. Mod. Phys., 71(3):463-512, 1999.

[5] L. P. Pitaevskii and S. Stringari. Bose-Einstein condensation. Clarendon Press, Oxford ; New York, 2003.

[6] A. J. Leggett. Bose-Einstein condensation in the alkali gases: Some fundamental concepts. Rev. Mod. Phys., 73(2):307-356, 2001.

[7] L.P. Pitaevskii. Zh. Eksp. Theor. Phys., 40:646, 1961.

[8] E.P. Gross. Structure of a quantized vortex in boson systems. Nuovo Cimento, 20(3):454-477, 1961.

[9] L.P. Pitaevskii. J. Math. Phys., 4:195, 1963.

[10] L. Erdös, B. Schlein, and H. T. Yau. Rigorous derivation of the Gross-Pitaevskii equation. Phys. Rev. Lett., 98(4):040404, 2007.

[11] E. H. Lieb and R. Seiringer. Proof of Bose-Einstein condensation for dilute trapped gases. Phys. Rev. Lett., 88(17):170409, 2002. 
[12] L. S. Cederbaum and A. I. Streltsov. Best mean-field for condensates. Phys. Lett. A, 318(6):564-569, 2003.

[13] O. E. Alon and L. S. Cederbaum. Pathway from condensation via fragmentation to fermionization of cold bosonic systems. Phys. Rev. Lett., 95(14):140402, 2005.

[14] P. W. Anderson. Absence of diffusion in certain random lattices. Phys. Rev., 109(5):1492, 1958.

[15] K. Ishii. Localization of eigenstates and transport phenomena in one-dimensional disordered system. Suppl. Prog, Theor. Phys., 53(53):77-138, 1973.

[16] P. A. Lee and T. V. Ramakrishnan. Disordered electronic systems. Rev. Mod. Phys., $57(2): 287-337,1985$.

[17] I. M. Lifshits, L. A. Pastur, and S. A. Gredeskul. Introduction to the theory of disordered systems. Wiley, New York, 1988.

[18] E. Abrahams, P. W. Anderson, D. C. Licciardello, and T. V. Ramakrishnan. Scaling theory of localization - absence of quantum diffusion in 2 dimensions. Phys. Rev. Lett., 42(10):673-676, 1979.

[19] T. Schwartz, G. Bartal, S. Fishman, and M. Segev. Transport and Anderson localization in disordered two-dimensional photonic lattices. Nature, 446(7131):52-55, 2007.

[20] H. Gimperlein, S. Wessel, J. Schmiedmayer, and L. Santos. Ultracold atoms in optical lattices with random on-site interactions. Phys. Rev. Lett., 95(17):170401, 2005.

[21] J. E. Lye, L. Fallani, M. Modugno, D. S. Wiersma, C. Fort, and M. Inguscio. Bose-Einstein condensate in a random potential. Phys. Rev. Lett., 95(7):070401, 2005.

[22] D. Clement, A. F. Varon, M. Hugbart, J. A. Retter, P. Bouyer, L. Sanchez-Palencia, D. M. Gangardt, G. V. Shlyapnikov, and A. Aspect. Suppression of transport of an interacting elongated Bose-Einstein condensate in a random potential. Phys. Rev. Lett., 95(17):170409, 2005.

[23] D. Clement, A. F. Varon, J. A. Retter, L. Sanchez-Palencia, A. Aspect, and P. Bouyer. Experimental study of the transport of coherent interacting matter-waves in a $1 \mathrm{D}$ random potential induced by laser speckle. New J. Phys., 8:165, 2006.

[24] L. Sanchez-Palencia, D. Clement, P. Lugan, P. Bouyer, G. V. Shlyapnikov, and A. Aspect. Anderson localization of expanding Bose-Einstein condensates in random potentials. Phys. Rev. Lett., 98(21):210401, May 2007.

[25] J. Billy, V. Josse, Z. C. Zuo, A. Bernard, B. Hambrecht, P. Lugan, D. Clement, L. SanchezPalencia, P. Bouyer, and A. Aspect. Direct observation of Anderson localization of matter waves in a controlled disorder. Nature, 453(7197):891-894, June 2008.

[26] C. Fort, L. Fallani, V. Guarrera, J. E. Lye, M. Modugno, D. S. Wiersma, and M. Inguscio. Effect of optical disorder and single defects on the expansion of a Bose-Einstein condensate in a one-dimensional waveguide. Phys. Rev. Lett., 95(17):170410, 2005.

[27] E. Akkermans, S. Ghosh, and Z. H. Musslimani. Numerical study of one-dimensional and interacting Bose-Einstein condensates in a random potential. J. Phys. B, 41(4):045302, 2008.

[28] T. Paul, P. Schlagheck, P. Leboeuf, and N. Pavloff. Superfluidity versus Anderson localization in a dilute Bose gas. Phys. Rev. Lett., 98(21):210602, 2007.

[29] A. R. Bishop. Fluctuation phenomena : disorder and nonlinearity. World Scientific, Singapore ; River Edge, NJ, 1995.

[30] K. O. Rasmussen, D. Cai, A. R. Bishop, and N. Gronbech-Jensen. Localization in a nonlinear disordered system. Europhys. Lett., 47(4):421-427, 1999.

[31] G. Kopidakis and S. Aubry. Intraband discrete breathers in disordered nonlinear systems. I. Delocalization. Physica D, 130(3-4):155-186, 1999.

[32] G. Kopidakis and S. Aubry. Discrete breathers and delocalization in nonlinear disordered systems. Phys. Rev. Lett., 84(15):3236-3239, 2000.

[33] D. L. Shepelyansky. Delocalization of quantum chaos by weak nonlinearity. Phys. Rev. Lett., 70(12):1787-1790, 1993.

[34] A. S. Pikovsky and D. L. Shepelyansky. Destruction of Anderson localization by a weak nonlinearity. Phys. Rev. Lett., 100(9):094101, 2008.

[35] M. Mulansky. Localization properties of nonlinear disordered lattices. Universität Potsdam, Diploma thesis, 2009. http://nbn-resolving.de/urn:nbn:de:kobv:517-opus-31469.

[36] H. Veksler, Y. Krivolapov, and S. Fishman. Phys. Rev. E, 2009. to appear.

[37] G. Kopidakis, S. Komineas, S. Flach, and S. Aubry. Absence of wave packet diffusion in disordered nonlinear systems. Phys. Rev. Lett., 100(8):084103, 2008. 
[38] M. I. Molina. Transport of localized and extended excitations in a nonlinear Anderson model. Phys. Rev. B, 58(19):12547-12550, 1998.

[39] S. Flach, D. Krimer, and Ch. Skokos. Universal spreading of wavepackets in disordered nonlinear systems. Phys. Rev. Lett., 102:024101, 2009.

[40] C. Skokos, D.O. Krimer, Komineas, and S. S. Flach. Delocalization of wave packets in disordered nonlinear chains. Phys. Rev. E, 79:056211, 2009.

[41] J. M. Combes and P. D. Hislop. Localization for some continuous, random hamiltonians in d-dimensions. J. Funct. Anal., 124(1):149-180, 1994.

[42] F. Germinet and S. De Bièvre. Dynamical localization for discrete and continuous random Schrödinger operators. Commun. Math. Phys., 194(2):323-341, 1998.

[43] F. Klopp. Localization for some continuous random Schrödinger-operators. Commun. Math. Phys., 167(3):553-569, 1995.

[44] A. Soffer and W.-M. Wang. Anderson localization for time periodic random Schrödinger operators. Commun. Partial Differ. Equ., 28(1-2):333-347, 2003.

[45] J. Bourgain and W. M. Wang. Anderson localization for time quasi-periodic random schrödinger and wave equations. Commun. Math. Phys., 248(3):429-466, 2004.

[46] W.-M. Wang. Logarithmic bounds on Sobolev norms for time dependent linear Schröinger equations. Comm. Part. Diff. Eq., 33(12):2164-2179, 2008.

[47] V. Nersesyan. Growth of Sobolev norms and controllability of Schrödinger equation. Commun. Math. Phys., 290:371-387, 2009.

[48] W.-M. Wang and Z. Zhang. Long time Anderson localization for nonlinear random Schrödinger equation. J. Stat. Phys., 134:953, 2009.

[49] C. Albanese and J. Fröhlich. Periodic-solutions of some infinite-dimensional hamiltoniansystems associated with non-linear partial difference-equations .1. Commun. Math. Phys., 116(3):475-502, 1988.

[50] C. Albanese, J. Fröhlich, and T. Spencer. Periodic-solutions of some infinite-dimensional hamiltonian-systems associated with non-linear partial difference-equations .2. Commun. Math. Phys., 119(4):677-699, 1988.

[51] C. Albanese and J. Fröhlich. Perturbation-theory for periodic-orbits in a class of infinite dimensional hamiltonian-systems. Commun. Math. Phys., 138(1):193-205, 1991.

[52] A. Iomin and S. Fishman. Localization length of stationary states in the nonlinear Schrödinger equation. Phys. Rev. E, 76(5):056607, 2007.

[53] S. Fishman, A. Iomin, and K. Mallick. Asymptotic localization of stationary states in the nonlinear Schrödinger equation. Phys. Rev. E, 78:066605, 2008.

[54] S. Fishman, Y. Krivolapov, and A. Soffer. On the problem of dynamical localization in the nonlinear Schrödinger equation with a random potential. J. Stat. Phys., 131(5):843-865, 2008.

[55] G. Benettin, J. Fröhlich, and A. Giorgilli. A Nekhoroshev-type theorem for Hamiltoniansystems with infinitely many degrees of freedom. Commun. Math. Phys., 119(1):95-108, 1988.

[56] M. Aizenman and S. Molchanov. Localization at large disorder and at extreme energies - an elementary derivation. Commun. Math. Phys., 157(2):245-278, 1993.

[57] R. del Rio, S. Jitomirskaya, Y. Last, and B. Simon. What is localization ? Phys. Rev. Lett., 75(1):117-119, 1995.

[58] R. del Rio, S. Jitomirskaya, Y. Last, and B. Simon. Operators with singular continuous spectrum .IV. Hausdorff dimensions, rank one perturbations, and localization. J. Anal. Math., 69:153-200, 1996.

[59] S. De Bièvre and F. Germinet. Dynamical localization for the random dimer Schrödinger operator. J. Stat. Phys., 98(5-6):1135-1148, 2000.

[60] F. Germinet and A. Klein. New characterizations of the region of complete localization for random Schrödinger operators. J. Stat. Phys., 122(1):73-94, 2006.

[61] S. Fishman, Y. Krivolapov, and A. Soffer. On the distribution of linear combinations of eigenvalues of the Anderson model. work in progress.

[62] M. Aizenman and S. Warzel. On the joint distribution of energy levels of random Schrödinger operators. J. Phys. A, 42:045201, 2009.

[63] A. Erdélyi. Asymptotic expansions. Dover, New-York, 1956. 
Physics Department, Technion - Israel Institute of Technology, Haifa 32000, IsRAEL.

E-mail address: fishman@physics.technion.ac.il

Physics Department, Technion - Israel Institute of Technology, Haifa 32000, IsRAEL.

E-mail address: evgkr@tx.technion.ac.il

Mathematics Department, Rutgers University, New-Brunswick, NJ 08903, USA.

E-mail address: soffer@math.rutgers.edu 